\title{
A revision of Peltariosilis Wittmer (Coleoptera: Cantharidae), a surprisingly diverse Amazonian radiation
}

\author{
Gabriel Biffi' \& Michael Geiser ${ }^{2}$
}

\author{
1 Universidade de São Paulo (USP), Museu de Zoologia (MZUSP). São Paulo, SP, Brasil. \\ ORCID: http://orcid.org/0000-0001-7532-3822. E-mail: biffigabriel@gmail.com \\ 2 Natural History Museum, Department of Life Sciences. London, United Kingdom. \\ ORCID: http://orcid.org/0000-0002-9278-2358. E-mail:m.geiser@nhm.ac.uk
}

\begin{abstract}
Peltariosilis Wittmer, 1952 is a South American Silinae genus characterised by the highly modified male pronotum and scutellum with a remarkable lamellar projection. Six species were previously recognised. The study of numerous specimens from previously unsampled localities and the revision of previously studied material shows that Peltariosilis is far more diverse than previously recognised. 15 species are here recognised as valid, nine of which are described as new: $P$. brancuccii, P. brunneoapicalis, P. cleidecostae, P. diversicollis, P. flavicornis, P. gracilicornis, P. major, P. orientalis and P. parviscutellaris spp. nov. A comparative study and new morphological terminologies are introduced for pronotum, scutellum and male genitalia. All Peltariosilis species are described and illustrated, including the first illustration of a female pronotum, and an identification key is provided. A map is given, including records of all known Peltariosilis, showing a distribution confined to the Amazonian subregion (Suriname, French Guiana, N Brazil, E Peru and NE Bolivia). The identity of the type species P. scutulata (Wittmer, 1952), hitherto considered as broadly distributed and highly variable, is addressed through study of their type series and additional specimens from widespread localities.
\end{abstract}

Key-Words. Amazon; Morphology; Neotropical; Silinae; Silis; Taxonomy.

\section{INTRODUCTION}

Silinae are the second largest subfamily of Cantharidae in terms of described species. They are found on all continents but particularly diverse in the tropics. One of the most remarkable features of this subfamily is the high diversity of complex sexually dimorphic structures found in the males, all presumed to be associated with pheromone glands and used during mating (Eberhard, 2006; Hsiao et al., 2015). In most Silinae genera, these male "modifications" are located on the pronotum and are highly diagnostic species characters. One of the most remarkable examples is Peltariosilis Wittmer, 1952 from the Amazonian region of South America, where not only the male pronotum is highly complex, but also the scutellum bears a remarkable modified projection, in the shape of an obliquely or perpendicularly rising lamella, sometimes with a forked apex. This kind of structure is unique within Cantharidae.

Using a very wide concept of the genus Silis Charpentier, 1825, Wittmer (1952) established the subgenus Peltariosilis for two species, Silis scutulata Wittmer, 1952 from Manaus, Brazil, and
S. guyanensis Pic, 1906 from French Guiana. Later, Wittmer (1966) described P. amapaensis from Amapá state, northern Brazil.

Constantin (2010a) assessed the morphology of the three known species and elevated Peltariosilis to genus rank, adding as diagnostic characters the long, stout mandibles with a tooth at their base, the very short and widely emarginated epistoma, the very thin, short and adpressed elytral pubescence, the pronotum with anterior and posterior projections, and characters of the aedeagus. Also, he provided an identification key and new distributional records for the species.

As a result of long-term prospections in French Guiana, Constantin (2017) revised Peltariosilis and doubled the number of known species describing P. mensaemontis, P. mitarakaemontis and P. lamellata, and presented reliable illustrations of diagnostic features and a new identification key. Also, the distribution of species was expanded, especially for P. scutulata, broadly recorded throughout the Amazonian part of Brazil (Amazonas, Pará and Acre states), Peru and Bolivia (Constantin, 2017; Constantin \& Chaboo, 2016). 
These recent advances prompted us to address two questions: With French Guiana now being relatively well explored, the known diversity in this country is far higher than in all adjacent areas, such as northern Brazil, and the existence of further species within the rest of the range of the genus becomes very likely. Furthermore, the highly restricted distributions of most species contrasts with the broad distribution of $P$. scutulata, which also shows considerable morphological variations across its range.

In this work, we investigated further material of Peltariosilis, mostly from previously unknown localities within the Amazonian forests and revisited previously studied specimens, resulting in the discovery of new species. We also re-examined the supposedly variable and widespread $P$. scutulata in more detail, including a comparative study of their genitalia, revealing that they actually belong to a number of clearly distinct species, mostly with more restricted distributions. This led us to address nomenclatural issues regarding the identity of $P$. scutulata, since its type series turned out to be composed of two different species, which do not fit the species concept adopted by Constantin (2017).

This surprisingly diverse fauna of Peltariosilis led us to re-evaluate the current diversity of the genus and to propose a new revision for it, describing new species, addressing nomenclatural issues, and presenting new identification key and distribution maps.

\section{MATERIAL AND METHODS}

Institutions cited along the text where the types and other examined specimens are deposited:

BMNH - Natural History Museum, London, United Kingdom; CEMT - Seção de Entomologia da Coleção Zoológica da Universidade Federal do Mato Grosso, Cuiabá, Brazil; CCo - Robert Constantin collection, SaintLô, France; CZMA - Coleção Zoológica do Maranhão, Caxias, Brazil; DZUP - Coleção de Entomologia Pe. Jesus Santiago Moure, Departamento de Zoologia da Universidade Federal do Paraná, Curitiba, Brazil; HNHM - Natural History Museum (Magyar Természettudományi Múzeum), Budapest, Hungary; INPA - Instituto Nacional de Pesquisas da Amazônia, Manaus, Brazil; MNHN Muséum national d'Histoire naturelle, Paris, France; MNRJ - Museu Nacional do Rio de Janeiro, Rio de Janeiro, Brazil; MPEG - Museu Paraense Emílio Goeldi, Belém, Brazil; MZUSP - Museu de Zoologia da Universidade de São Paulo, São Paulo, Brazil; NHMB - Naturhistorisches Museum Basel, Basel, Switzerland; NMPC - Národní Muzeum, Prague, Czech Republic.

Label data are given verbatim. Information for each specimen is within quotation marks, labels are separated by double slashes (//), and single slashes (/) separate lines on the same label. All specimens of new species bear our red holotype or paratype label with taxon name. Complementary information is given within square brackets.
General morphological terminology used in the descriptions and dissection methods follow Brancucci (1980) and Constantin (2015). A new terminology is proposed for pronotal and scutellar structures. Until today, there is no universally accepted terminology for aedeagus structures within the subfamily Silinae. Therefore, the terminology from Constantin (2015) is used here to facilitate the comparison with other Neotropical genera of Silinae (e.g., Constantin, 2015, 2017; Biffi \& Constantin, 2018).

Photographs were taken on a Zeiss AxioCam MRc 5 video camera attached to a Carl Zeiss Discovery stereomicroscope or with Canon EOS Rebel T3i camera with Canon MP-E 65 mm macro-lens and StackShot macro-rail. Multi-focus images were combined with Zerene Stacker version 1.04. Illustrations were produced via camera lucida attached to stereomicroscope. Illustrations and photographs were edited in Adobe Photoshop CS6 and Adobe Illustrator CS6. The species are organised in the text and plates by similarity in order to enable an easier morphological comparison. For species that were recently described and well-illustrated (see Constantin, 2017) we provide only a differential diagnosis.

The general distribution map was created with Quantum GIS version 2.18.7-1 (available at http://www. qgis.org). Localities in the map are approximate localities obtained from specimens' labels and published literature sources (Constantin, 2010a, 2017).

\section{RESULTS}

\section{Peltariosilis Wittmer, 1952 \\ (Figs. 1-17)}

Silis (Peltariosilis) Wittmer, 1952: 203, Delkeskamp 1977: 286; Constantin 2010a: 41.

Peltariosilis, Constantin 2010b: 15, 2017: 61.

Type species: Silis (Peltariosilis) scutulata Wittmer, 1952, by original designation.

Diagnosis (Males): Clypeus slightly projected, anterior margin with an arched notch; mandibles with a minute tooth near base; pronotum variously modified, anterior margin broadly arched, sides with spines, lobes and projections; scutellum projected anteriorly in a broad lamella or narrow fork; anterior fore tarsal claw with a small rounded basal lobe, mid and hind anterior tarsal claw with small basal cleft. Peltariosilis is distinguished from other genera of Silinae by the unique scutellum, bearing an obliquely or perpendicularly rising lamella, sometimes with a forked apex.

Description: Head, thorax, wings and abdomen dark brown to black; antennae ranging from entirely pale yellow to entirely dark brown; elytra usually dark brown to black, sometimes lighter near lateral margins and suture, or broadly yellowish light brown; pronotum and scutellum pale yellow to light orange brown, sometimes trans- 
lucent. Body regularly covered with dense very short and fine adpressed pubescence and sparse longer setae on antennae and abdomen.

Males: Head (Fig. 1A) (including eyes) broad, nearly as wide as pronotum, ventral part retracted; gula long, gular sutures broadly separated; occipital region and vertex broadly convex, frons flattened; clypeus broadly fused to frons, frontoclypeal suture absent; clypeus broad, slightly prominent, anterior margin with an arched notch; labrum membranous, very reduced, barely distinct in frontal view; genae very short, indistinct in front of eyes. Eyes prominent, bulging, rounded, nearly as long as wide, broadly separated. Antennal insertions broadly separated; antennae usually filiform, sometimes with swollen, rounded antennomeres. Mandibles (Fig. 1A) long, stout, apex acute and sharp, smooth or with a minute tooth near base, lateral margins with rather long and dense pubescence. Maxillae weakly sclerotised; stipes short and broad, galea and lacinia membranous, densely setose; maxillary palpi longer than stipes; palpomeres I-III similar in shape, elongate, IV securiform. Labium weakly sclerotised, submentum membranous, palpi 3-segmented, palpomere III securiform. Pronotum (Figs. 2, 10) variously modified; anterior margin broadly arched, sides forming a frontolateral lobe (fl); sides behind frontolateral lobe parallel to slightly convergent posteriorly, distalmost part forming a broad or narrow laterobasal lobe (lbl) directed up and medially; angles (ang) of laterobasal lobes acute, obtuse, rounded or forming an unciform spine; apex of laterobasal lobe entire or divided into dorsal (df) and ventral flaps (vf). Dorsal surface forming two lateral bumps divided by a median groove (mg), a pair of dorsal projections (dp) that may be broadly rounded or sharp, razor blade shape, sometimes merging with laterobasal lobe; a deep basal groove (bg) behind laterobasal lobes; a pair of glandular pores anteriorly (gp). Scutellum (Figs. 2, 12) projected (sp) upwards and anteriorly in a broad trapezoidal lamella (Figs. 2C, 12A-F) or in a narrow stem divided apically in a bifid, well-defined fork (Figs. 2F, 12G-O). Elytra smooth, long, completely concealing the wings and abdomen; lateral sides parallel to divergent, wider posteriorly; apex rounded to nearly truncate; lateral and sutural margins not forming distinct borders. Thorax weakly sclerotised, metaventrite convex. Legs small, slender, without strong modifications in size, shape or pubescence; tibiae with one pair of tiny apical spurs; fourth tarsomere with a transversal slit at base; anterior fore tarsal claws with a small rounded basal lobe; anterior mid hind tarsal claws with a small straight basal cleft. Wings (Fig. 3) short and broad, radial cell 2R closed, veins $r$ and $r-m$ not coinciding in the meeting point of $\mathrm{Rr}$, Rr prolonged anteriorly beyond the meeting point of $r-m$, vein cu-a weakly sclerotised, barely visible, anal vein (A) not divided. Abdomen weakly sclerotised, flattened, short and broad; glandular pores very small, barely visible, not prominent, located near the distal margin of tergites; tergite VIII (Fig. 13) long and broad, with lateral margins straight to arched, glandular pores shallow to rather prominent, distal margin variously notched; ventrites I-VI very wide, distal margin straight; ventrite (i.e., visible sternite) VII (Fig. 14) with a very deep longitudinal incision, dividing it into two halves. Aedeagus (Figs. 4, 15-17): ventral plaque of tegmen (tg) lateral, flattened, very elongate, nearly as long as internal sac, variously shaped, straight, curved or sinuous, apex acute, rounded or truncate; parameres ( $p$ ) dorsally, united at base and moderately to broadly divergent from mid length, elongate, broadly curved dorsally, apex acute; median lobe $(\mathrm{ml})$ thinly membranous, translucent, tubular around internal sac and a series of sclerites; internal sac (is) membranous, very elongate, slender, straight to slightly curved dorsally, apex sometimes slightly swollen; a series of sclerites variable in number, position and shape: one pair of median dorsal sclerite (mds) that can be separated, fused medially or totally fused forming one single median sclerite, dorsal, very elongated, straight to curved ventrally, apex acute; one pair of paramedian dorsal sclerites (pds) robust, strongly sclerotised, flattened, usually curved or inclined dorsally, apex acute, gradually or abruptly narrowed, sometimes with scratches or projecting teeth; one pair of ventral sclerites (vs) very long, slender, pressed against ventrolateral wall of internal sac, apex acute, usually directed laterally; one pair of lateral sclerites (Is) present or absent.

Female: Similar to males in colouration and general morphology, differing from males on the base of dimorphic features, such as the antennae not swollen at middle antennomeres, pronotum (Fig. 11) wider and flattened, without strong modifications, anterior margin less arched, frontolateral and laterobasal lobes rounded, short, not pronounced or recurved; scutellum flattened, not projected anteriorly; pygidium more transverse; abdominal ventrite VII entire, not longitudinally divided, not distinctive among species.

Distribution: French Guiana, Suriname, Brazil, Peru, Bolivia (Fig. 18).

\section{List of Peltariosilis species}

P. amapaensis (Wittmer, 1966)

P. brancuccii sp. nov.

P. brunneoapicalis sp. nov.

P. cleidecostae sp. nov.

$P$. diversicollis sp. nov.

P. flavicornis sp. nov.

P. gracilicornis sp. nov.

P. guyanensis (Pic, 1906)

P. lamellata Constantin, 2017

P. major sp. nov.

P. mensaemontis Constantin, 2017

P. mitarakaemontis Constantin, 2017

P. orientalis sp. nov.

P. parviscutellaris sp. nov.

P. scutulata (Wittmer, 1952) 


\section{A key to Peltariosilis species (males)}

1. Scutellar projection lamellar (Figs. 12A-F), broader at base and narrower at apex; angles of laterobasal lobe forming strong spikes or unciform spines (Figs. 10A-F)

1'. Scutellar projection forked apically (Figs. 12G-0), very slender or narrower at middle, with divergent apices; angles of laterobasal lobe acute, obtuse or rounded (Figs. 10G-0)

2(1). Dorsal projections of pronotum small, sharp, razor-blade like (Fig. 10F) ...

.P. major sp. nov.

2. $\quad$ Dorsal projections of pronotum (Figs. 10A-E) broad and rounded .

$3\left(2^{\prime}\right)$. Angles of laterobasal lobe forming long, straight to slightly curved spikes (Fig. 10D) ..

lamellata Constantin, 2017

3'. Angles of laterobasal lobe forming strong, curved, unciform spines...

$4\left(3^{\prime}\right)$. Laterobasal lobes of pronotum mostly concealed by dorsal projections

4'. Sides of laterobasal lobes of pronotum visible, not completely concealed by dorsal projections (Fig. 10E).

5(4). Apex of dorsal projections of pronotum acute, pointing posteriorly (Fig. 10A)

5. $\quad$ Apex of dorsal projections of pronotum broadly rounded, directed postero-laterally (Fig. 10B-C).

$6\left(5^{\prime}\right)$. Apex of laterobasal lobe of pronotum forming a sharp hook directed internally (Fig. 10B)

6. Apex of laterobasal lobe of pronotum broad, truncate, directed posteriorly (Fig. 10C)

guyanensis (Pic, 1906)

$7\left(1^{\prime}\right)$. Antennae entirely yellow (Figs. $\left.6 \mathrm{~A}, \mathrm{C}\right)$

7. $\quad$ Antennae entirely dark brown to black or with two distal antennomeres yellow ......

8(7). Well-defined scutellar fork, with acute apices diverging from the middle (Fig. 12l); antennomeres thick; aedeagus (Figs. 16l-L): ventral plaque of tegmen straight, apex sickle like, apex of ventral sclerites slightly divergent P. flavicornis sp. nov.

8'. $\quad$ Scutellar fork flattened, apices slightly divergent from apical third (Fig. 12G); antennomeres slender; aedeagus (Figs. 16A-D): ventral plaque of tegmen curved, apex rounded with an acute pointing tip, apex of ventral sclerites broadly divergent P. gracilicornis sp. nov.

$9\left(7^{\prime}\right)$. Scutellar projection not forming well-defined fork, narrow, sides parallel or gradually widening apicad, apices slightly projected (Figs. 12M-0).............. 10

9'. Scutellar projection forming well-defined fork, much narrower at middle or apical third and distinctly broader at apex, apices acute, well projected (Figs. 12H-L)

10(9). Elytra testaceous yellow anteriorly with broad apical light brown spot (Fig. 7C); scutellum as in Fig. 120 ...

P. brunneoapicalis sp. nov.

10'. Elytra black, slightly lighter at lateral margins and along suture; scutellum different (Figs. 12M, N)

11(10). Dorsal projections of pronotum very wide and sharp, directed laterally (Fig. 10M)

11. Dorsal projections of pronotum small, rounded, slightly projected (Fig. 10N)

P. parviscutellaris sp. nov.

12(9'). Apices of scutellar fork parallel, pointing upwards (Figs. 12K-L) P. mitarakaemontis Constantin, 2017

12. $\quad$ Apices of scutellar fork divergent (Figs. 12H, J).......

3(12). Scutellar fork small (Fig. 12K); distal margin of last ventrite projecting at middle (Fig. 14H); distal margin oflast tergite with a deep and broad median notch (Fig. 13H); aedeagus (Figs. 17A-D): ventral plaque of tegmen parallel at the base, convergent at the apex, gradually narrowing at apex and slightly curved internally, with a dorsally curved acute tip

P. cleidecostae sp. nov.

13'. Scutellar fork large (Fig. 12L); distal margin of last ventrite barely rounded (Fig. 14I); distal margin of last tergite with a shallow and narrow median notch (Fig. 13I); aedeagus (Figs. 17E-H): ventral plaque of tegmen convergent at the base, parallel at the apex, strongly narrowed at apex, with a sharp acute tip curved dorsally.

P. brancuccii sp. nov.

14(12'). Dorsal projections of pronotum narrow laterally, not reaching lateral margins of pronotum (Fig. 10J); aedeagus (Figs. 16M-P): paramedian dorsal sclerites slightly curved dorsally, apex narrowed and divergent, pointing laterally. P. orientalis sp. nov.

14. Dorsal projections of pronotum wide laterally, reaching lateral margins of pronotum (Fig. 10H); aedeagus (Figs. 16E-H): paramedian dorsal sclerites slightly curved dorsally and abruptly folded, with apex sharp, pointing dorsally. P. scutulata (Wittmer, 1952)

Peltariosilis amapaensis (Wittmer, 1966)

(Figs. 1A, 3, 5A, 8A-D, 10A, 11A, 12A, 13A, 14A, 15A-D)

Silis (Peltariosilis) amapaensis Wittmer, 1966: 414; Delkeskamp 1977: 286.

Peltariosilis amapaensis, Constantin 2010b: 16, 2017: 61.

Type material examined: HOLOTYPE ơ (MZUSP 31226): "HOLOTYPUS // Ex - coleção / M. Alvarenga // Oiapoque / Amapá - Brasil /V . 1959 / M. Alvarenga col. // Peltariosilis / amapaensis / Wittm./ det. W.Wittmer // MZSP 31226" (Figs. 8A-D). PARATYPES (7 specimens): same data as holotype (4 o', MZUSP 31227-31230, 3 o', NHMB).

Other material examined: "Oiapoque / Amapá, Brasil / V . 1959 / Alvarenga col. // Peltariosilis / amapaensis / (Wittmer, 1966) / det. G. Biffi 2018 // MZUSP 31231" (1 o",
MZUSP 31231); "Serra Lombard / Limão / 19.8-1961 // BRASIL, AP / J. \& B. Bechyné // Peltariosilis / amapaensis / (Wittmer, 1966) / det. G. Biffi 2018" (2 o", 1 \&, MPEG); "Serra Lombard / Limão / 20.8-1961 // BRASIL, AP / J. \& B. Bechyné // Peltariosilis / amapaensis / (Wittmer, 1966) / det. G. Biffi 2018" (2 o", MPEG); "Serra Lombard / Limão / 28.8-1961 // BRASIL, AP / J. \& B. Bechyné // Peltariosilis / amapaensis / (Wittmer, 1966) / det. G. Biffi 2018" (1 o", MPEG); "Serra Lombard / Olemarque / 10.VIII.1961 // Brasil, AP / J. \& B. Bechyné" (1 o", BMNH); "Guyane, station Nourages [Nouragues] / saut Pararé / $4^{\circ} 01^{\prime} \mathrm{N}, 52^{\circ} 41^{\prime} \mathrm{W}$, 120m / 23.XI.2009, piège vitre / P.Dalens, S. Brûlé, E.Poirie [Poirier] // Peltariosilis / amapaensis / Wittmer, 1966 / R. Constantin det.2016" (1 o", CCo).

Diagnosis: Antennomeres IX-XI narrower than preceding ones. Pronotum with very short laterobasal lobe, one 
lateral unciform spine, posterior portion with an acute pointing projection posteriorly; prediscal projections convex, broadly rounded, not meeting dorsal edge of laterobasal lobe. Scutellar projection short, trapezoidal, lamellar, slightly narrowed at apex, distal margin sinuated, with three shallow rounded projections. Peltariosilis amapaensis is similar to P. lamellata, P. guyanensis, P. mensaemontis and $P$. diversicollis sp. nov. by the general aspect of pronotum. It differs from these species by the posterior margin of dorsal projections of pronotum with an acute pointing tip.

Re-description: Head entirely black; maxillae and labium light brown, slightly darker on distal palpomeres; antennae mostly dark brown, slightly lighter at base of each antennomere, antennomeres I-II orangish to light brown; pronotum and scutellum light orange brown, slightly translucent; elytra dark brown, lighter at lateral margins; legs light orange brown, darker on the apex of tibiae and tarsi; thorax and abdomen dark brown, darker on last abdominal ventrite and tergite.

Male: (Fig. 5A) Antennae short; antennomere I elongate, slightly wider apically, II short, slightly wider apically, III-IV nearly cylindrical, V-VIII flat at ventral surface and globose at dorsal surface, IX-XI slender, filiform. Pronotum (Fig. 10A): frontolateral lobe narrow; laterobasal lobe very short, mostly concealed under dorsal projections, angles with lateral unciform spines, apex with an acute pointing projection posteriorly; dorsal projections very wide, internal margin concave, lateral and posterior margins broadly rounded with an acute pointing tip. Scutellum (Fig. 12A) with a short trapezoidal lamellar projection, wide at base, slightly narrowed at apex, distal margin sinuated, with three shallow rounded projections. Elytra divergent at lateral margins, wider posteriorly; apex truncate. Abdominal tergite VIII (Fig. 13A) subtrapezoidal, lateral margins convergent posteriorly, wider anteriorly; glandular pores slightly prominent; distal margin sinuous, with a small, shallow median notch. Ventrite VII (Fig. 14A) wide, lateral margins sinuous, broadly arched and wider anteriorly, convergent and constricted posteriorly; posterior margin arched; internal margins of median incision nearly parallel. Aedeagus (Fig. 15A-D): ventral plaque of tegmen lateroventral, shorted than internal sac, flattened laterally, wider medially, apex acute, sinuous, gradually curved internally, apex curved externally; parameres broadly fused at base, divergent at apex, slightly flattened dorsoventrally, regularly curved dorsally, tips rounded; median lobe membranous, translucent; internal sac very elongate, directed dorsally; median dorsal sclerite absent; paramedian dorsal sclerites short and narrow, divergent, straight, apex slightly acute, directed dorsally; ventral sclerites very elongated, almost as long as internal sac, slender, straight, apex acute, slightly curved laterally; lateral sclerites as long as paramedian dorsal sclerites, robust, strongly sclerotised.

Female: General appearance and colouration similar to males; antennae slender, simple, middle antennom- eres not distinctly globose; pronotum (Fig. 11A) without strong modifications; frontolateral lobe smaller, posterior margin arched; laterobasal lobe broad, oblique; dorsal projections absent; median and basal grooves shallow; ventrite VII with a small median notch on distal margin.

Distribution: French Guiana, Brazil (Amapá state) (Fig. 18).

\section{Peltariosilis guyanensis (Pic, 1906) (Figs. 5B, 8E-I, 10B, 12B)}

Silis guyanensis Pic, 1906: 92; Delkeskamp 1939: 179; Blackwelder 1945: 367.

Silis (Peltariosilis) guyanensis, Wittmer, 1952: 203; Delkeskamp 1977: 286; Constantin 2010a: 41.

Peltariosilis guyanensis, Constantin 2010b: 16; 2017: 61.

Type material examined: LECTOTYPE ơ (MNHN), new designation: "Cayene // SYNTYPE // Silis / guyanensis / Pic 1906 / R.Constantin det. 2009 // Peltariosilis / guyanensis / (Pic) / det.W.Wittmer" (Fig. 8E-I). PARALECTOTYPE o" (NHMB): "DÉCEMBRE // GUYANE FRANÇSE / StLaurent du Maroni / COLLECTION LE MOULT // cotype // Naturhistorisches / Museum Basel / Coll. W. Wittmer // CANTHARIDAE / CANTH00018168". PARALECTOTYPE o" (NHMB): "AVRIL // GUYANE / NOUVEAU CHANTIER / BAS-MARONI // cotype // Silis / guyanensis / Pic // Naturhistorisches / Museum Basel / Coll. W. Wittmer // CANTHARIDAE / CANTH00017764".

Other material examined: "FRENCH GUIANA / Montagne des Chevaux / Window trap; xi.2009; $4^{\circ} 36^{\prime} 37.6^{\prime \prime} \mathrm{N} 52^{\circ} 16^{\prime} 44.5^{\prime \prime} \mathrm{W} /$ altitude $225 \mathrm{~m}$ a.s.l. // BMNH(E) / 2010-62" (2 o", BMNH); "Zanderij I, Boven / Para Dist.SURINAM / April 191927 // Cornell U. / Lot 760 / Sub 28 // Naturhistorisches / Museum Basel / Coll. W. Wittmer" (1 o', NHMB); "Zanderij I, Boven / Para Dist. SURINAM / April 261927 // Cornell U. / Lot 760 / Sub 41 // Naturhistorisches / Museum Basel / Coll. W. Wittmer" (1 o', NHMB).

Diagnosis: Antennae dark brown, yellow at apex of antennomere Xl; head entirely black; pronotum and scuteIlum orange, slightly translucent; elytra black, except for undefined lighter lateral margins and sutures; legs light yellow, except for tarsi, light brown; thorax and abdomen dark brown. Male (Fig. 5B): Antennomeres IX-XI narrower than preceding ones. Pronotum (Fig. 10B): anterior margin broadly arched; frontolateral lobes narrow, projected laterally; sides behind frontolateral lobe convergent posteriorly; laterobasal lobe with dorsal flap long, strongly curved, hook-like projection directed medially and ventral flap forming a sharp lamellar projection; dorsal projections broadly rounded directed posteriorly and laterally. Scutellum (Fig. 12B) with a short trapezoidal lamellar projection, wide at base, slightly narrowed at apex, distal margin almost straight, with two slightly projecting tips at angles. Peltariosilis guyanensis differs 
from $P$. mensaemontis by the apex of dorsal flap of laterobasal lobe of pronotum, forming a sharp hook directed internally.

Distribution: Suriname, French Guiana (Fig. 18).

Remarks: Pic (1906) described this species from an unspecified number of males from "Cayenne". We found three male specimens at MNHN and NHMB, all with different locality data, which we here consider part of the original type series. This includes a specimen at NHMB cited as a non-type by Constantin (2010a), which however bears a "cotype" label in Pic's handwriting. The male syntype at MNHN, in Pic's own collection, is here designated as the lectotype.

\section{Peltariosilis mensaemontis Constantin, 2017 (Figs. 5C, 10C, 12C)}

Peltariosilis mensaemontis Constantin, 2017: 62.

Type material examined: PARATYPE $\sigma^{\prime \prime}$ (CCo): "Guyane Française / MontTabulaire-Itoupé $/ 3^{\circ} 01^{\prime} 19^{\prime \prime} \mathrm{N}-53^{\circ} 05^{\prime} 03^{\prime \prime} \mathrm{W}$ / alt.800m,auto'LT PinkLED / 25.XI.2014,SEAG \& Dalens // PARATYPE / Peltariosilis / mensaemontis / Constantin i.I. / R. Constantin des. 2016".

Diagnosis: Head and antennae black, ventral face of antennomere I-Il yellow to light brown, pronotum orangish, scutellum reddish, elytra black, legs yellow, apical two thirds of tibiae and four basal tarsomeres brown. Male (Fig. 5C): Antennae long, antennomeres IV-VIII swollen, subcylindrical, IX-XI slender, narrower than preceding ones. Pronotum (Fig. 10C): anterior margin strongly arched, broadly continuous with frontolateral lobes; sides behind frontolateral lobe concave; laterobasal lobe with dorsal flap broad, apex truncate; dorsal projections broad, divergent, distal margin rounded, directed posterolaterally. Scutellum (Fig. 12C) with vertical trapezoidal lamellar projection, slightly longer than wide at base. Peltariosilis mensaemontis differ from $P$. guyanensis by the dorsal flap of laterobasal lobe of pronotum broad, truncate at apex.

Distribution: French Guiana (Fig. 18).

\section{Peltariosilis Iamellata Constantin, 2017 (Figs. 5D, 10D, 12D)}

Peltariosilis lamellata Constantin, 2017: 61.

Type material examined: PARATYPE o" (CCo): "Guyane fr., massif Mitaraka / sommet en cloche; savane roche / 20.VIII.2015, auto-LTgemlight / $2^{\circ} 13^{\prime} 41^{\prime \prime} \mathrm{N}-54^{\circ} 28^{\prime} 5^{\prime \prime} \mathrm{W}$ 310 m / P.Dalens,S.Brûlé,E.Poirier,F.Robin // La Planète Revisitée-MNHN / PNI Guyane 2015-APA-973-1 /collecte, tri \& préparation / Société entomologique AntillesGuyane // Paratype pelt. / lamellata / Paratype / lamellata".
Diagnosis: Head and antennae black, except for antennomere I and ventral face of II, pronotum orangish, scutellum reddish, legs orangish yellow, distal half of tibiae and four basal tarsomeres infuscate. Male (Fig. 5D): Antennae long, antennomeres V-VIII swollen, ovoid, IX-XI slender, narrower than preceding ones. Pronotum (Fig. 10D): anterior margin arched, broadly continuous with frontolateral lobes; sides behind frontolateral lobe concave; laterobasal lobe not projected laterally, curved posteriorly; angles of laterobasal lobe forming long, acute spikes; dorsal projections broad, distal margin rounded, directed dorsally. Scutellum (Fig. 12D) with vertical trapezoidal lamellar projection, as long as wide at base, apex truncate. Peltariosilis lamellata differ from $P$. amapaensis by the angles of laterobasal lobes of pronotum forming long, slightly curved spikes, and dorsal projection shorter, posterior margin rounded, directed dorsally.

Distribution: French Guiana (Fig. 18).

\section{Peltariosilis diversicollis sp. nov. (Figs. 2A-C, 5E, 10E, 12E, 13B, 14B, 15E-H)}

Type series: HOLOTYPE ơ (MPEG): "Rio Calçoene / 2-81961 // Brasil, AP / J. \& B. Bechyné" (Fig. 5E). PARATYPES (2 specimens): "Porto Platon / 21-7-1961 // Brasil, AP / J. \& B. Bechyné" (1 o", MPEG); "R. CASSIPORÉ / Vila Velha / 14 / 15-9-1961 // Brasil, AP / J. \& B. Bechyné// MZUSP 31232" (1 o', MZUSP 31232).

Diagnosis: Pronotum with laterobasal lobe flattened, slit posteriorly into dorsal and posterior flaps with acute pointing projections posteriorly, and lateral unciform spine; dorsal projections flattened, broadly rounded, meeting dorsal edge of laterobasal lobe; sides of laterobasal lobes visible laterally, not concealed under dorsal projections. Scutellum with a short trapezoidal lamellar projection, wider than long, wide at base. Peltariosilis diversicollis sp. nov. differs from P. lamellata by the curved, unciform spines at the angles of laterobasal lobes, from P. major sp. nov. by the broad dorsal projections of pronotum, and from $P$. amapaensis, $P$. guyanensis and $P$. mensaemontis by the lateral sides of laterobasal lobes not concealed under dorsal projections.

Description: Head entirely black; maxillae and labium light orange brown, slightly darker on distal palpomeres; antennae mostly dark brown, slightly lighter at dorsal surface of antennomeres IV-VIII; antennomeres I-II and apex of XI orangish to light brown; pronotum and scutellum light orange brown, slightly translucent; elytra dark brown, lighter at lateral margins; legs light orange brown, darker on the apex of tibiae and tarsi; thorax and abdomen dark brown, darker on last abdominal ventrite and tergite.

Male: (Fig. 5E): Antennae short; antennomere I elongate, slightly wider apically, II short, cylindrical, III-IV nearly 

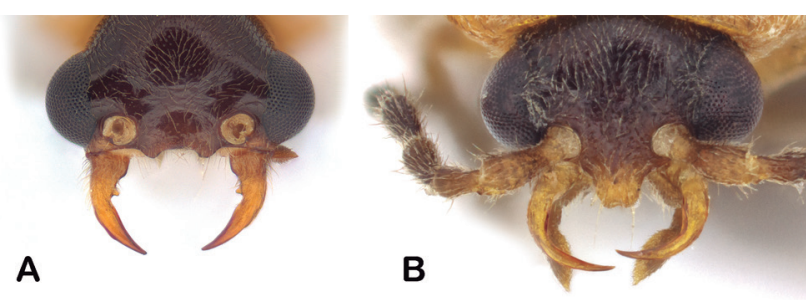

Figure 1. Head of Peltariosilis species, dorsal. (A) P. amapaensis (Wittmer, 1966), male. (B) P. parviscutellaris sp. nov., female.

cylindrical, V-VIII flat at ventral surface and slightly globose at dorsal surface, IX-XI slender, filiform. Pronotum (Fig. 10E): anterior margin broadly arched, continuous with frontolateral lobes; frontolateral lobes broad, projected laterally; laterobasal lobes with one lateral unciform spine, flattened, slit posteriorly into dorsal and posterior flaps with acute pointing projections posteriorly; dorsal projections flattened, broadly rounded, meeting dorsal edge of laterobasal lobe. Scutellum (Fig. 12E) with a short trapezoidal lamellar projection, wider than long, wide at base, narrow at apex, distal margin notched. Elytra divergent at lateral margins, wider posteriorly; apex truncate. Abdominal tergite VIII (Fig. 13B) long and broad, lateral margins broadly rounded, wider medially; glandular pores not protruding; distal margin sinuous, with a small, shallow median notch. Ventrite VII (Fig. 14B) wide, lateral margins sinuous, broadly arched anteriorly, wider medially, constricted posteriorly; posterior margin arched; internal margins of median incision nearly parallel. Aedeagus (Fig. 15E-H): ventral plaque of tegmen lateroventral, shorter than internal sac and ventral sclerites, flattened laterally, wider medially, sinuous, broadly curved, apex acute and curved dorsally; parameres broadly fused at base and divergent towards apex, apex strongly curved dorsally, rounded with small acute tip; median lobe membranous, translucent; internal sac tubular, very elongate, straight, membranous, slightly sclerotised at apex; median dorsal sclerite absent; one pair of small, well-sclerotised lateral sclerites concealed behind parameres; paramedian dorsal sclerites elongated, nearly straight, broader at base and gradually narrowing at apex, apex acute; ventral sclerites very elongated, almost as long as internal sac, slender, straight, apex acute, divergent, slightly curved laterally.

Female: Unknown.

Etymology: The epithet diversicollis is formed from the Latin words diversus (different) + collum ("neck", prothorax), referring to the distinct shape of prothorax in this species.

Distribution: Brazil (Amapá state) (Fig. 18).

\section{Peltariosilis major sp. nov. (Figs. 4E-H, 5F, 10F, 12F, 13C, 14C, 15I-L)}

Type series: HOLOTYPE ơ (INPA): "BRASIL, Amapá, Serra do Navio, / Estrada Lagoa Azul, / $0^{\circ} 52^{\prime} 52^{\prime \prime} \mathrm{N}-51^{\circ} 58^{\prime} 49^{\prime \prime} \mathrm{W}$, / Varredura, 18.iv.2014, J.T. / Câmara, A. Plant \& J.A. Rafael // Peltariosilis / n. sp. / det. M. Geiser 2018" (Fig. 5F).

Diagnosis: Pronotum with laterobasal lobe flattened, apex curved downwards, with acute lateral spine; dorsal projections very small, sharp, distal margins sinuous, with acute lateral apex acute, pointing posteriorly;
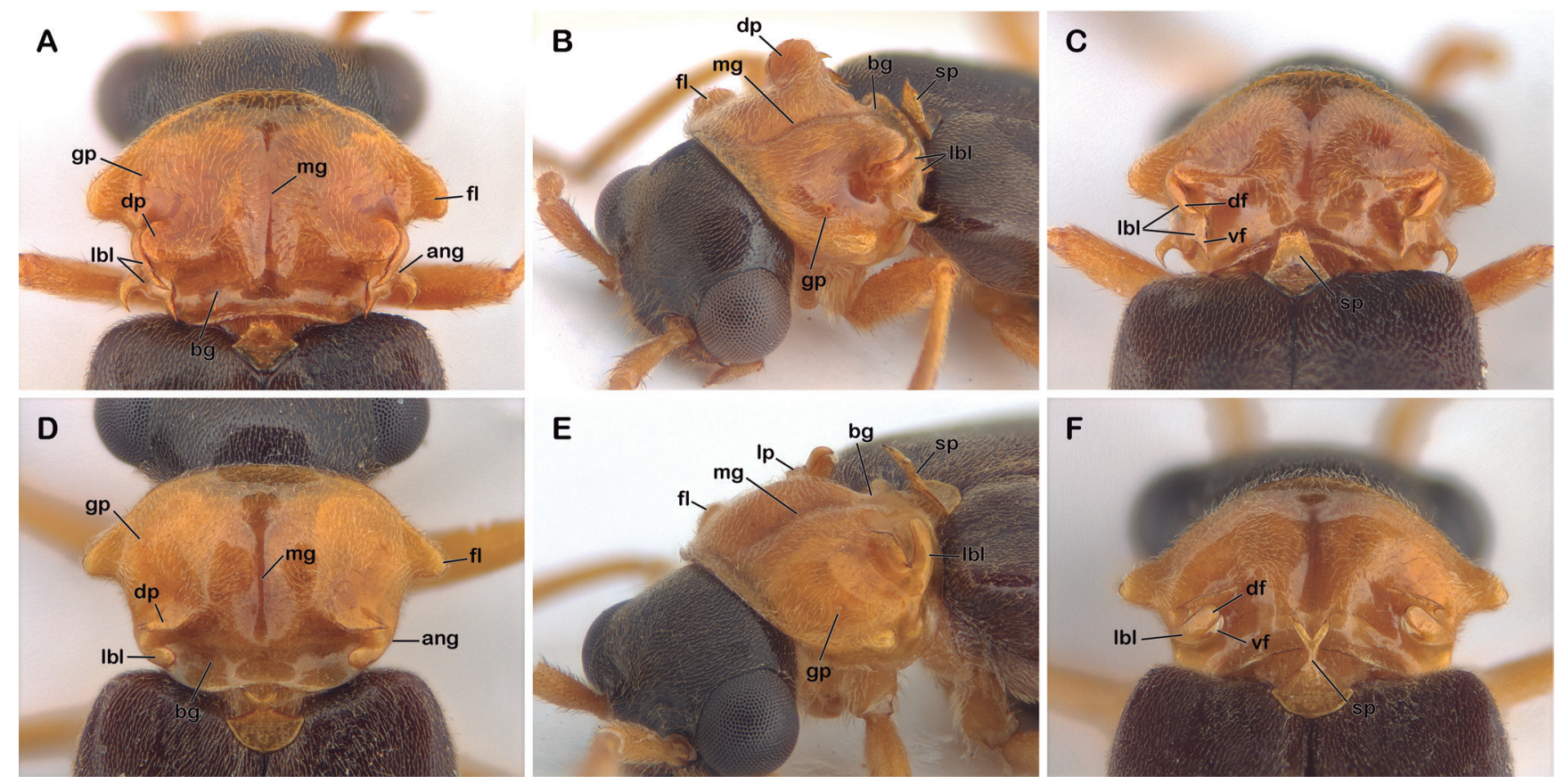

Figure 2. Morphology of pronotum of Peltariosilis species, males. (A-C) P. diversicollis sp. nov. (D-F) P. flavicornis sp. nov. Legends: ang: angle of laterobasal lobe; bg: basal groove; dp: dorsal projection; fl: frontolateral lobe; gp: glandular pore; lbl: laterobasal lobe (df: dorsal flap, vf: ventral flap); mg: median groove; sp: scutellar projection. 


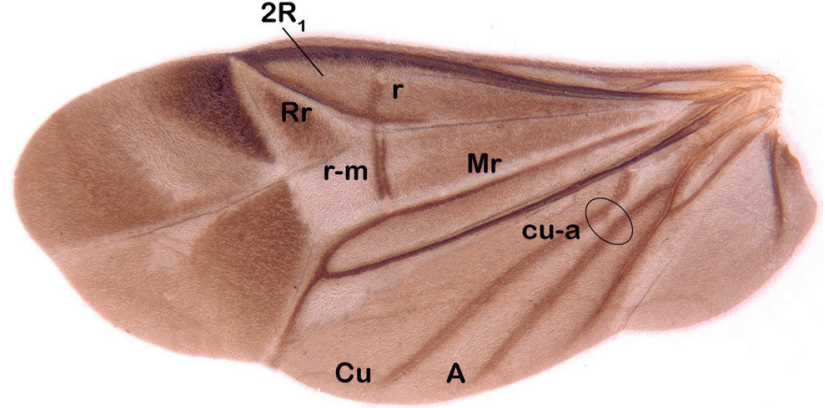

Figure 3. Wing of Peltariosilis amapaensis (Wittmer, 1966).

scutellar projection lamellar, long, triangular, nearly symmetrical, regularly narrowing apically. Peltariosilis major sp. nov. differs from all other species by the very small dorsal projections of pronotum and by laterobasal lobes flattened dorsally with apex acute curved downwards.

Description: Head mostly black, slightly lighter at anterior margin of clypeus; maxillae and labium testaceous yellow; mandibles light brown, darker apically; antennae mostly black, antennomeres I-II orange brown, X-XI dark brown; pronotum and scutellum light orange brown, slightly translucent; elytra dark brown, lighter at lateral margins; legs light orange brown; thorax and abdomen dark brown.

A

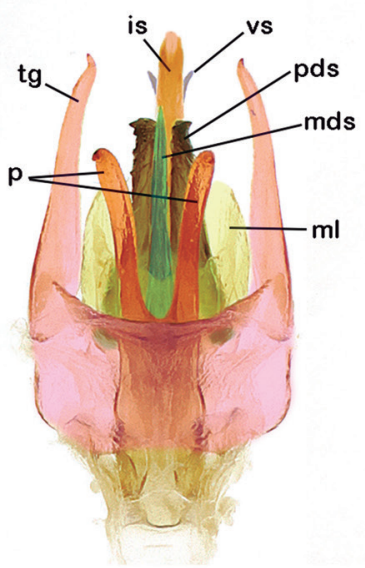

E

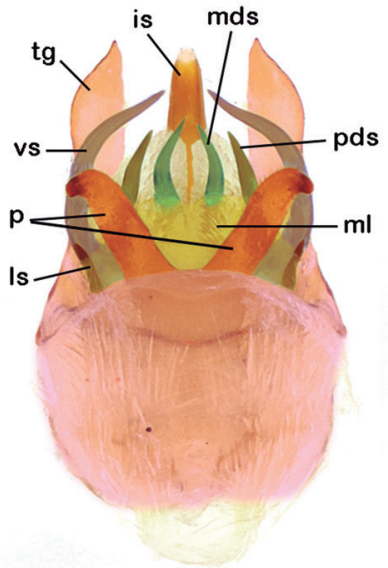

B

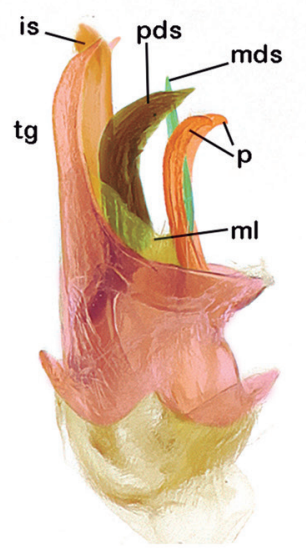

$\mathbf{F}$

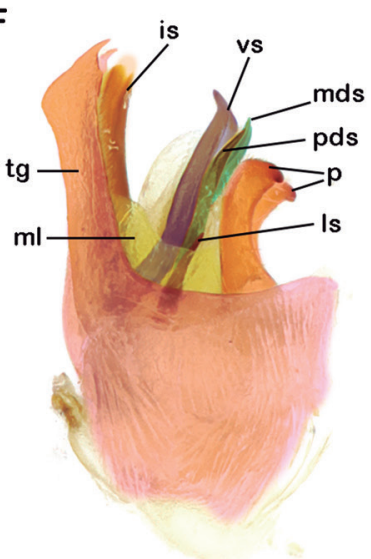

Male: (Fig. 5F): Antennae short; antennomere I elongate, slightly wider apically, II short, cylindrical, III-VIII nearly fusiform, slightly swollen, IX-XI slender. Pronotum (Fig. 10F): anterior margin broadly arched, continuous with frontolateral lobes; laterobasal lobe with one small acute lateral spine, dorsal surface flattened, posterior margin sharp, oblique, apex acute, strongly curved downwards; dorsal projections very small, posterior margin sharp, sinuous, with small acute lateral spine pointing posteriorly. Scutellum (Fig. 12F) with a long, nearly symmetrical, triangular lamellar projection, regularly narrowing apically. Elytra nearly parallel, slightly wider posteriorly; apex truncate. Abdominal tergite VIII (Fig. 13C) very broad, trapezoidal, lateral margins broadly rounded, wider medially; glandular pores not protruding; distal margin concave, with a small, shallow rounded median notch. Ventrite VII (Fig. 14C) wide, lateral margins slightly convergent posteriorly, wider anteriorly; posterior margin arched; internal margins of median incision nearly parallel, halves broadly separated. Aedeagus (Fig. 15I-L): ventral plaque of tegmen lateroventral, as long as internal sac, flattened laterally, broad, apex oblique with an acute dorsal pointing tip; parameres fused at base and broadly divergent apically and curved dorsally, apex rounded with small tip pointing dorsally; median lobe membranous, translucent; internal sac tubular, very elongate, slightly curved dorsally, moderately sclerotised, apex membranous; one

C

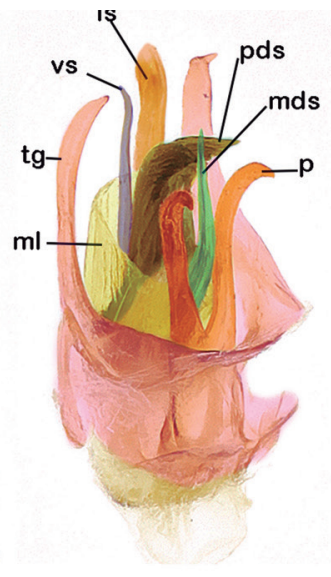

G

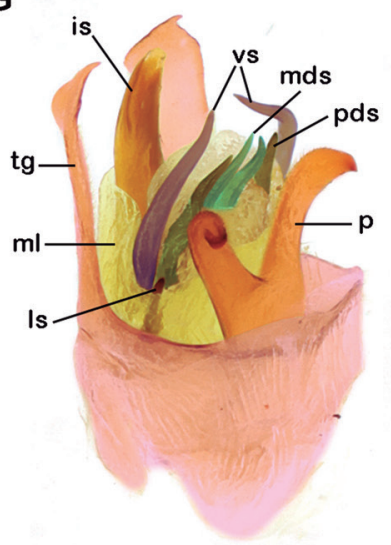

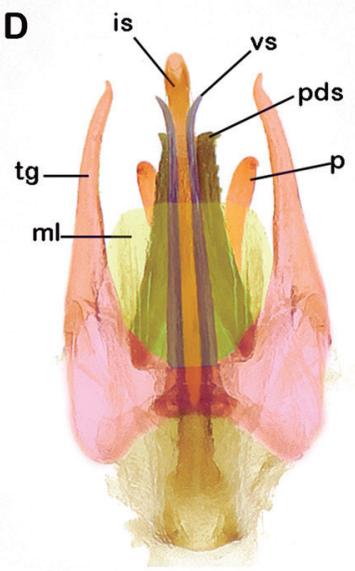

D

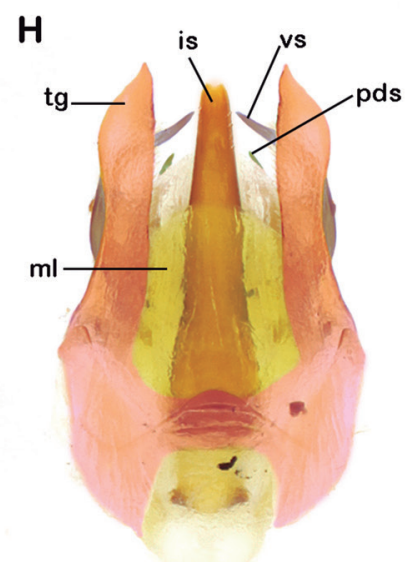

Figure 4. Morphology of aedeagus of Peltariosilis species. (A-D) P. cleidecostae sp. nov. (E-H) P. major sp. nov. Legends: is: internal sac; Is: lateral sclerite; mds: median dorsal sclerite; ml: median lobe; $p$ : parameres; $p d s$ : paramedian dorsal sclerites; tg: tegmen; vs: ventral sclerite. 
pair of median dorsal sclerites sinuous, divergent at base than convergent at apex, apex acute, slightly curved ventrally; one pair of short, acute, sclerotised lateral sclerites; paramedian dorsal sclerites elongate, slightly curved internally narrowing at apex, flanking median dorsal sclerites; ventral sclerites not flanking internal sac, parallel to dorsal sclerites and paramedian dorsal sclerites, very elongated, slender, sinuous, curved internally, apex acute, convergent, directed ventrally.

Female: Unknown.

Etymology: The specific epithet major (Latin for "larger") refers to the larger size of the species in comparison with the others.

Distribution: Brazil (Amapá state) (Fig. 18).

\section{Peltariosilis gracilicornis sp. nov. (Figs. 6A, 10G, 12G, 13D, 14D, 16A-D)}

Type series: HOLOTYPE ơ (INPA): "BRA, Amazonas, Novo / Aripuanã, lago Xadá / 05¹5'39"S 6042'32"W / iv.2005 // Em luz mista e BLB / F. Xavier, F.Godoi / \& A. Lourido leg." (Fig. 6A).

Diagnosis: Antennae entirely testaceous yellow, slender, not swollen; pronotum and scutellum orange brown. Pronotum with low, small dorsal projections; laterobasal lobes slightly curved, directed dorsally and posteriorly, apex rounded, not slit. Scutellum lamellar, broad, with a wide forked projection; apices divergent, latero-anteriorly. Peltariosilis gracilicornis sp. nov. differs from all other species by the slender testaceous-yellow antennae, without tumescence, the shape of laterobasal bobes and dorsal projections of pronotum and, especially by unique shape of scutellar projection.

Description: Head black, except anterior margin of clypeus, orange brown; mandibles, labium, maxillae and palpi testaceous to light brown; antennae entirely testaceous yellow; pronotum and scutellum orange brown, slightly translucent; elytra dark brown, lighter at lateral margins and along suture; legs testaceous yellow to light orange brown slightly darker at apex of tibiae and tarsi; thorax and abdomen dark brown, except near the distal and lateral margins of abdominal ventrites.

Male: (Fig. 6A): Antennae slender; antennomere I elongate, slightly wider apically, II short, cylindrical, III-XI cylindrical, not distinctly swollen. Pronotum (Fig. 10G): anterior margin arched, continuous with frontolateral lobes; laterobasal lobes without lateral spines, forming a barely distinct angle or cusp; laterobasal lobe slightly curved, directed posteriorly and dorsally, apex rounded, not distinctly split; dorsal projections low, transverse, directed postero-dorsally, forming blunt lateral peaks barely reaching sides of pronotum. Scutellum (Fig. 12G) lamellar, with a long forked projection anteriorly; scute-
Ilar projection gradually narrowed at middle and widened toward the apex, forming a broad apical fork with divergent apices pointing latero-anteriorly. Elytra nearly parallel, apex rounded. Abdominal tergite VIII (Fig. 13D) subquadrate, nearly as long as wide, lateral margins sinuous, wider medially; glandular pores not protruding; distal margin with a shallow rounded median notch. Ventrite VII (Fig. 14D) wide, lateral margins parallel anteriorly and convergent posteriorly, apex rounded; internal margins slightly arched, divergent, halves broadly separated. Aedeagus (Figs. 16A-D): ventral plaque of tegmen lateroventral, shorter than internal sac and ventral sclerites, flattened laterally, broad, lateral margins curved, apex rounded with an acute dorsal pointing tip; parameres fused at base and parallel apically, apex curved with small tip pointing dorsally; median lobe membranous, translucent; internal sac tubular, very elongate and broad, more sclerotised apically; one pair of paramedian dorsal sclerites straight and convergent at base, with divergent apices curved laterodorsally, apex acute; lateral sclerites indistinct, median dorsal sclerite elongated, narrowing apically, apex acute; ventral sclerites ventrally, partially flanking internal sac, very elongated, slender, slightly convergent apically, than apices divergent, acute, convergent, directed laterally.

Female: Unknown.

Etymology: The specific epithet gracilicornis is formed from the Latin words gracilis (slender) + cornis ("horn", antenna), referring to the slender antennae.

Distribution: Brazil (Amazonas state) (Fig. 18).

\section{Peltariosilis scutulata (Wittmer, 1952) (Figs. 6B, 9A-D, 10H, 12H, 13E, 14E, 16E-H)}

Silis (Peltariosilis) scutulata Wittmer, 1952: 203, Delkeskamp 1977: 286.

Peltariosilis scutulata, Constantin 2010b: 16, 2017: 63.

Type material examined: HOLOTYPE ("Allotype") $\sigma^{7}$ (NHMB):"AmazonasMauans / Parko II 1941 // S. / scutulata /Wittm. / det. W. Wittmer // Allotypus // Naturhistorisches / Museum Basel / Coll. W. Wittmer // CANTHARIDAE / CANTH00018355" (Figs. 9A-E). PARATYPE ơ (NHMB): "Amazonas / B. Constant / Parko.2.1941 // Paratypus // 3 // Naturhistorisches / Museum Basel / Coll. W. Wittmer // CANTHARIDAE / CANTH00018445" (Figs. 9F-J).

Other material examined: "BRASIL. Roraima, Caracaraí / (Vila Caicubi, trilha Pupunha) / 0059'27,1"S, 62 05'55.8"W / 10.ix.2011 / Biffi, G. \& Prado, L.R. col. // MZUSP 31233"(1 o", MZUSP 31233); "BRASIL: Amazonas, Novo Airão, / Rod. AM 352, Km-68, lgarapé / Mato Grosso, 17-31.VIII.2016, / $02^{\circ} 48^{\prime} 58^{\prime \prime} \mathrm{S}, 60^{\circ} 55^{\prime} 18^{\prime \prime} \mathrm{W}$. / Malaise peq próximo igarapé / J.A. Rafael \& F.F. Xavier leg." (1 0', CEMT); "2395 // Est. Am1. Km104 / Mn. Am. Brasil / Em 5/68 / Col-Vários" (1 o", INPA); "Tapuruquara / Rio Negro, AM / 26-27.XI.1962 / J. Bechyné 
col. // Convênio / DZSP- Goeldi // MZUSP 31234" (1 $0^{\text {, }}$ MZUSP 31234); "Rio Cauaburi / AM, Brasil / 9.XII.1962 / J. Bechyné col. // MZUSP 31235" (1 o", MZUSP 31235).
Diagnosis: Antennae dark brown, except antennomeres I-II and X-XI, testaceous yellow to light orange brown; pronotum and scutellum orange brown; elytra dark brown,

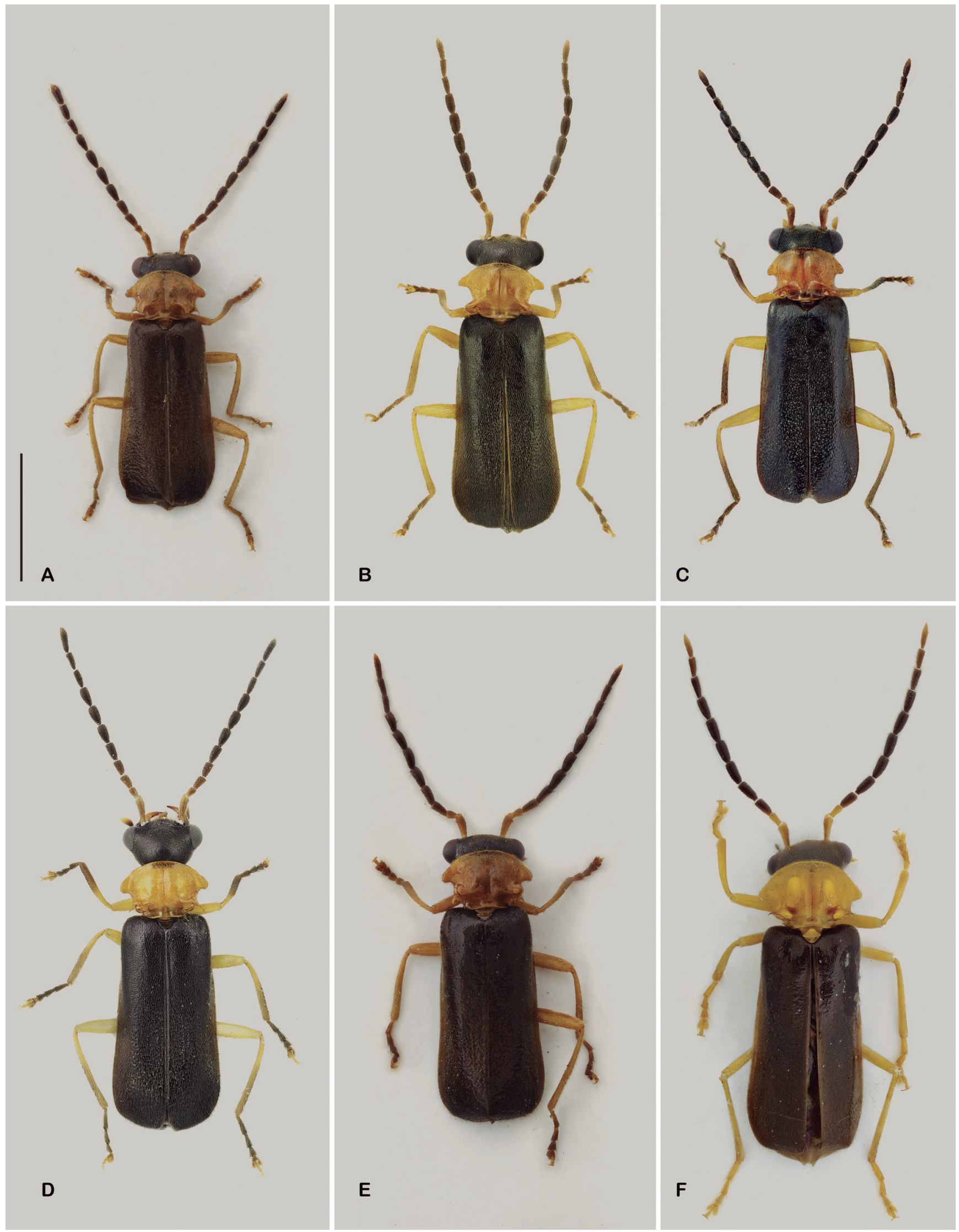

Figure 5. Habitus of Peltariosilis species. (A) P. amapaensis (Wittmer, 1966). (B) P. guyanensis (Pic, 1906). (C) P. mensaemontis Constantin, 2017. (D) P. lamellata Constantin, 2017. (E) P. diversicollis sp. nov. (F) P. major sp. nov. All figures in the same scale. Scale bar $=2.0 \mathrm{~mm}$. 
lighter at lateral margins and along suture. Antennomeres $\mathrm{X}$-XI narrower than preceding ones. Pronotum with low, sharp dorsal projections; laterobasal lobes straight, di- rected dorsally and posteriorly, apex rounded, swollen. Scutellum with a long forked projection; apices parallel, pointing anteriorly. Peltariosilis scutulata is a member of

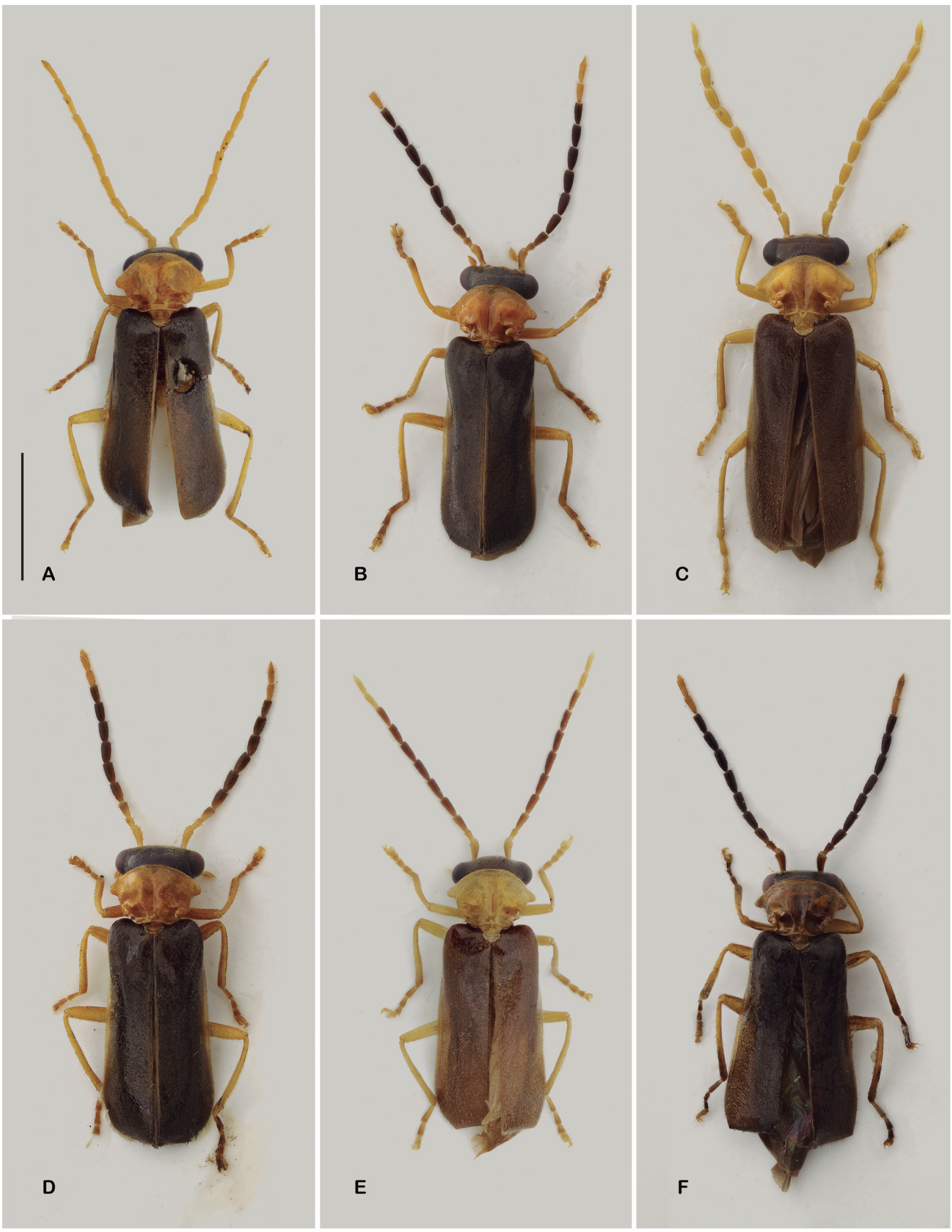

Figure 6. Habitus of Peltariosilis species. (A) P. gracilicornis sp. nov. (B) P. scutulata (Wittmer, 1952). (C) P. flavicornis sp. nov. (D) P. orientalis sp. nov. (E) P. cleidecostae sp. nov. (F) P. brancuccii sp. nov. All figures in the same scale. Scale bar $=2.0 \mathrm{~mm}$. 


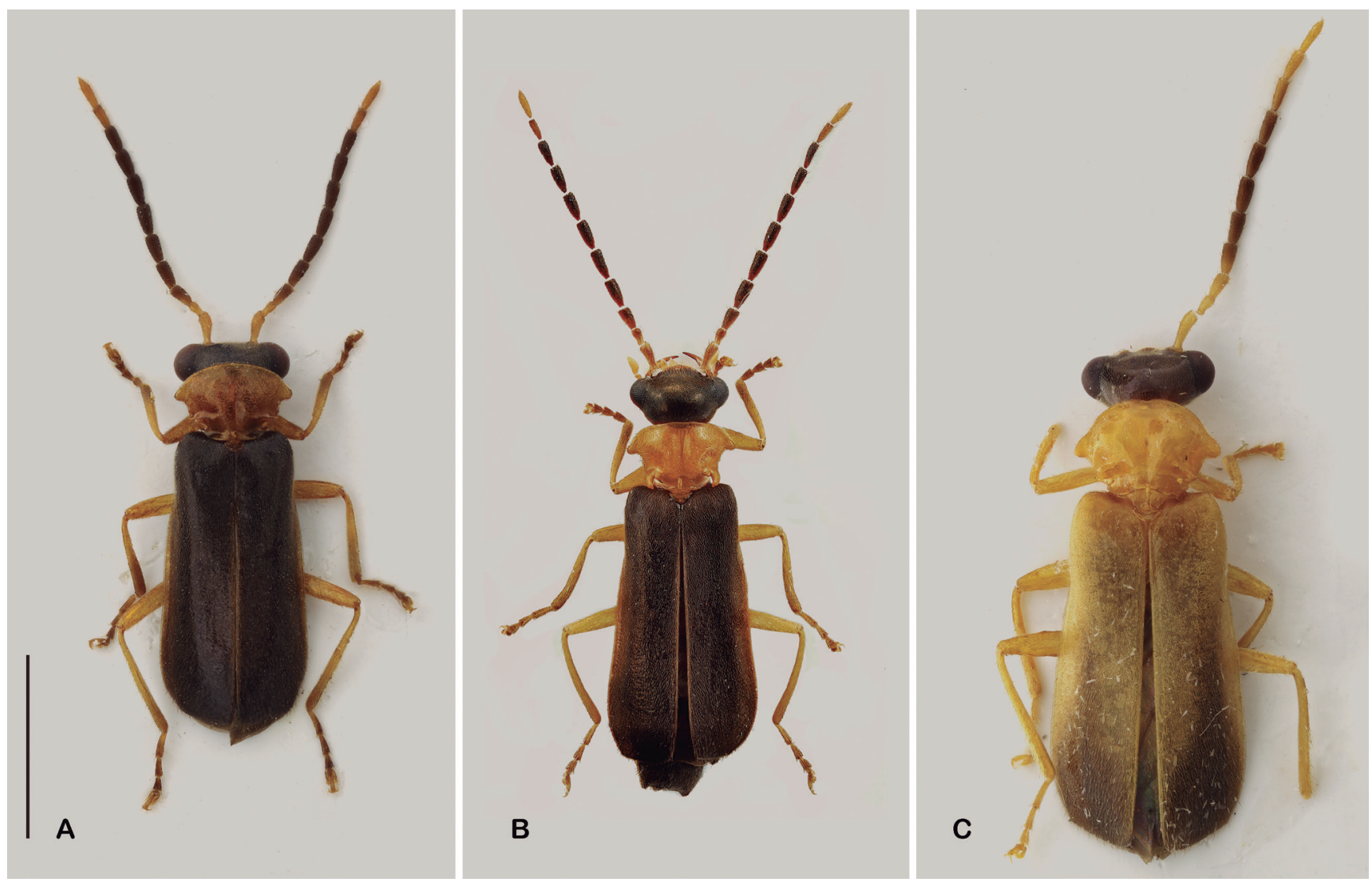

Figure 7. Habitus of Peltariosilis species. (A) P. parviscutellaris sp. nov. (B) P. mitarakaemontis Constantin, 2017. (C) P. brunneoapicalis sp. nov. All figures in the same scale. Scale bar $=2.0 \mathrm{~mm}$.

a group of species with somewhat similar pronotum and scutellar projections. It differs from $P$. mitarakaemontis, $P$. brunneoapicalis sp. nov. and P. parviscutellaris sp. nov. by the robust apices of scutellar fork, from $P$. cleidecostae sp. nov. and P. brancuccii sp. nov. by the rounded apex of laterobasal lobes; from $P$. gracilicornis sp. nov. and P. flavicornis sp. nov. by the mostly dark brown antennae, and from $P$. orientalis sp. nov. by the width of dorsal projections of pronotum, reaching lateral margins.

Re-description: Head black, except anterior margin of clypeus, orange brown; mandibles, labium, maxillae and palpi testaceous to light brown; antennae mostly dark brown, except antennomeres I-II, ventral face of III, and $\mathrm{X}$-XI testaceous yellow to light orange brown; pronotum and scutellum orange brown, slightly translucent; elytra dark brown, lighter at lateral margins and along suture; legs testaceous yellow to light orange brown slightly darker at apex of tibiae and tarsi; thorax and abdomen dark brown, except near the distal and lateral margins of abdominal ventrites.

Male: (Fig. 6B): Antennae long; antennomere I elongate, slightly wider apically, II short, slightly wider apically, III-IX nearly cylindrical, narrower proximally, flat at ventral surface and globose at dorsal surface, IX-XI narrower, slender, filiform. Anterior margin of clypeus with a broad arcuate concave notch. Pronotum (Fig. 10H): anterior margin broadly arched; frontolateral lobe narrow, projected laterally, sides behind frontolateral lobe slightly convergent; angles of laterobasal lobe rounded or barely obtuse; laterobasal lobe straight, directed posteriorly and dorsally, apex swollen, rounded, apparently split, forming juxtaposed dorsal and ventral flaps; apex of ventral flap with a short membranous projection; dorsal projections low, transverse, directed postero-dorsally, distal margin arcuate, forming an inner acute peak and lateral peaks barely reaching sides of pronotum. Scutellum (Fig. $12 \mathrm{H}$ ) with a long forked projection anteriorly; scutellar projection gradually narrowed, elongate and abruptly widened, forming apical fork with arched sides; apices of fork divergent, pointing latero-anteriorly. Elytra nearly parallel, apex rounded. Abdominal tergite VIII (Fig. 13E) subquadrate, lateral margins broadly arched, wider medially, distal margin with a shallow median notch. Ventrite VII (Fig. 14E) wide, lateral margins broadly arched, wider anteriorly, convergent posteriorly; posterior margins straight laterally, with a low rounded projection internally. Aedeagus (Figs. 16E-H): ventral plaque of tegmen lateroventral, elongated, longer than internal sac and sclerites, flattened laterally, curved internally, narrow, wider at apex, apex broadly rounded with a dorsally curved acute tip; parameres fused at base and slightly divergent, regularly curved dorsally, apex narrowed or rounded with a small acute tip; median lobe membranous, translucent; internal sac elongated, narrow, straight, nearly as long as ventral, median and paramedian dorsal sclerites; one single median sclerite, elongated, narrow, apex acute; paramedian dorsal sclerites robust, strongly sclerotised, elongated, slightly curved dorsally and abruptly folded, 
with apex sharp, pointing dorsally; ventral sclerites slender, shorter than internal sac, apex acute, slightly divergent; lateral sclerites indistinct.

Distribution: Brazil (Roraima and Amazonas states) (Fig. 18).

Remarks: Type series of S. (P.) scutulata: Wittmer (1952) described only the male of Silis (Peltariosilis) scutulata from a type series composed of a holotype (from MNRJ) and an "allotype" (from Wittmer's own collection, now housed at NHMB) from "Brasilien, Manaus (Amazonas) 2.1941 leg. Parko". Both specimens, located at NHMB, are males labelled as "allotype" and "paratype" - and not holotype. The "allotype" bears a locality label "Amazonas Mauans [lapsus for Manaus]" (Fig. 9E), whereas the "paratype" bears a locality label "Amazonas, B. [Benjamin] Constant" (Fig. 9J). The reason why the type specimens of S. scutulata are labelled as "paratype" and "allotype" is still unknown, but one of them actualy might comprise the holotype. The specimen labelled as "allotype" is here considered as the actual holotype of $S$. $(P$.) scutulata, given that that is the only specimen bearing a label with the type locality according to the original description. Fortunately, since the original description, this specimen had never been returned to the MNRJ, thus being saved from the fire that destroyed nearly the entire museum in September, 2018 (Kury et al., 2018).

On the identity of $P$. scutulata: In the two most recent papers on the genus (Constantin \& Chaboo, 2016; Constantin, 2017), this species was reported to be widely distributed within the Amazonian forests, from French Guiana and Pará state (Brazil) to Bolivia and Peru. With the limited amount of material available per locality, the diagnosis of the species was based only on external characters.

Now, our more extensive material and the examination of the male genitalia revealed clear differences between the populations in various parts of Amazonia, which lead us to the conclusion that $P$. scutulata sensu Constantin needs to be treated as a whole complex of species. What added to the confusion was the fact that Wittmer's own type series already represents two different species, with the paratype from Benjamin Constant belonging to a different, undetermined species. The remaining specimens identified as $P$. scutulata by Wittmer himself turned out to belong to three additional species (P. cleidecostae sp. nov., $P$. flavicornis sp. nov. and $P$. brancuccii sp. nov.). It appears that both Wittmer and Constantin interpreted the differences in pronotum structure, scutellar projection and antennal colouration as individual variability, but with our more extensive material some of these character turned out to be diagnostic for the different species.

With this in mind, we have to assume that the specimen illustrated by Constantin (2017) most likely doesn't belong to $P$. scutulata either, but at the moment we are not sure which species to assign it to. In our restricted sense, $P$. scutulata is a species found only in parts of Amazonian Brazil, notably around Manaus. Some records from other areas mentioned by Constantin (2017) are still pending re-examination.

\section{Peltariosilis flavicornis sp. nov.} (Figs. 2D-F, 6C, 10I, 11B, 12I, 13F, 14F, 16I-L)

Type series: HOLOTYPE ơ (NHMB): "Soil Zoological Exp. / Bolivia, Guayaramerin // Beni/outskirts of town / BALOGH, MAHUNKA, ZICSI // No. 405 / lamping / 24.XI.1966.". PARATYPES (198 specimens): "Soil Zoological Exp. / Brasil, Manaus /Amazonas / $20 \mathrm{~km}$ s of city / BALOGH, MAHUNKA, ZICSI // No. 360 / singled material / 13.XI.1966 // P. scutulata / Wittm. / det. Wittmer" (2 o", HNHM); "Soil Zoological Exp. / Bolivia, Guayaramerin // Beni/outskirts of town / BALOGH, MAHUNKA, ZICSI // No. 377 / lamping / 18.XI.1966." (1 o', NHMB, 3 o', HNHM);"Soil Zoological Exp./ Bolivia, Guayaramerin // Beni/200 m from river/ BALOGH, MAHUNKA, ZICSI // No. 377/ lamping / 18.XI.1966." (3 o", HNHM); "Soil Zoological Exp. / Bolivia, Guayaramerin // Beni/outskirts of town / BALOGH, MAHUNKA, ZICSI // No. 391 / lamping / 22.XI.1966. // Peltariosilis / scutulata / Wittm. / det. W. Wittmer" (2 o", NHMB, 23 o", HNHM); "Soil Zoological Exp. / Bolivia, Guayaramerin // Beni/ outskirts of town / BALOGH, MAHUNKA, ZICSI // No. 399 / lamping / 23.XI.1966." (88 o", HNHM, 8 o", MZUSP, $6 \sigma^{\prime \prime}$, BMNH, $20^{\circ}$, NMPC, $20^{\prime}$, MNHN, $20^{\circ}$, INPA, $20^{\prime \prime}$, MPEG, $2 \sigma^{\circ}$, CEMT, $2 \sigma^{\circ}$, CZMA, $2 \sigma^{\circ}$, DZUP, $2 \sigma^{\prime \prime}$, CCo, $\left.1 \sigma^{\prime \prime}, \mathrm{NHMB}\right) ;$ "Soil Zoological Exp. / Bolivia, Guayaramerin // Beni/outskirts of town / BALOGH, MAHUNKA, ZICSI // No. 405 / lamping / 24.XI.1966." (2 o", NHMB, 1 o", BMNH, 7 o", HNHM); "Soil Zoological Exp. / Bolivia, Guayaramerin // Beni/ outskirts of town / BALOGH, MAHUNKA, ZICSI // No. 417 / lamping / 29.-30.XI.1966." (1 o', NHMB, 3 o", HNHM); "Soil Zoological Exp. / Bolivia, Guayaramerin // Beni/ outskirts of town / BALOGH, MAHUNKA, ZICSI // No. 424 / lamping / 1.XII.1966." (1 ๔0, NHMB, 10 o", HNHM); "Soil Zoological Exp. / Bolivia, Guayaramerin // Beni/outskirts of town / BALOGH, MAHUNKA, ZICSI // No. 429 / lamping / 2.XII.1966. // Peltariosilis / scutulata / Wittm. / det. W. Wittmer" (1 o", NHMB, 3 o", HNHM); "Soil Zoological Exp. / Bolivia, Guayaramerin // Beni/outskirts of town / BALOGH, MAHUNKA, ZICSI // No. 429 / lamping / 2.XII.1966." (1 ơ', NHMB); "Soil Zoological Exp. / Bolivia, Guayaramerin // Beni/15 km W of town / BALOGH, MAHUNKA, ZICSI // No. 434/ lamping / 3.XII.1966." (3 o", HNHM); "Soil Zoological Exp. / Bolivia, Guayaramerin // Beni/outskirts of town / BALOGH, MAHUNKA, ZICSI // No. 442 netting/ in the grass/ 5.XII.1966." // P. scutulata / Wittm. / det. Wittmer" (1 o", HNHM); "Soil Zoological Exp. / Bolivia, Guayaramerin // Beni/outskirts of city [sic] / BALOGH, MAHUNKA, ZICSI // No. 443 netting/ from water/ 5.XII.1966." (1 o", HNHM); "Soil Zoological Exp. / Bolivia, Guayaramerin // Beni/outskirts of town/ BALOGH, MAHUNKA, ZICSI // No. 445/ lamping/ 5.XII.1966." (1 o", HNHM); "Guayaramerin / PANDO-BOLIVIA / XII-956, Prosen // 21998 // Naturhistorisches / Museum Basel / Coll. W. Wittmer" (1 o", 1 \%, NHMB); "Soil Zoological Exp. / BRASIL,Manaus /Amazonas // 20 km S of city / BALOGH, MAHUNKA, ZICSI // No. 360 / singled material 
/ 13.XI.1966" (2 ơ, NHMB, 1 ఠ", BMNH); "BRASIL. MT, Nova Mutum / Fazenda Buriti / 04 - 14.i.2002 - Luz / HF Mendes Col." (2* MZUSP 31242-31243).

Diagnosis: Antennae entirely pale yellow; pronotum and scutellum orange yellow; elytra dark brown, slightly lighter at lateral margins. Antennomeres X-XI slightly narrower than preceding ones, middle antennomeres not distinctly swollen. Pronotum with low, sharp dorsal projections; laterobasal lobes curved, directed dorsally and posteriorly, apex rounded, divided in two flaps. Scutellum with a long, forked projection; apices acute, divergent, pointing latero-anteriorly. Peltariosilis flavicornis sp. nov. is similar to $P$. gracilicornis sp. nov. by the entirely pale yellow antennae. They differ by the scutellar projection much narrower at middle in $P$. flavicornis sp. nov.

Description: Head black, except anterior margin of clypeus, dark brown; antennae, labium, maxillae, palpi and base of mandibles pale yellow, apex of mandibles light brown; pronotum and scutellum orange brown, slightly translucent; elytra dark brown, lighter at lateral margins; legs entirely testaceous yellow to light orange yellow; thorax and abdomen dark brown.

Male: (Fig. 6C): Antennae rather long; antennomere I elongate, slightly wider apically, II short, slightly wider apically, III-IX nearly oblong, narrower proximally, not distinctly swollen, slightly flattened dorsoventrally, IX-XI narrower, slender. Anterior margin of clypeus with a broad arcuate concave notch. Pronotum (Fig. 10I): anterior margin broadly arched; frontolateral lobe narrow, projected laterally, sides behind frontolateral lobe slightly convergent; angles of laterobasal indistinct; laterobasal lobe curved, directed posteriorly and dorsally, apex rounded, split, forming juxtaposed dorsal and ventral flaps; apex of ventral flap with a short membranous projection; dorsal projections low, transverse, directed
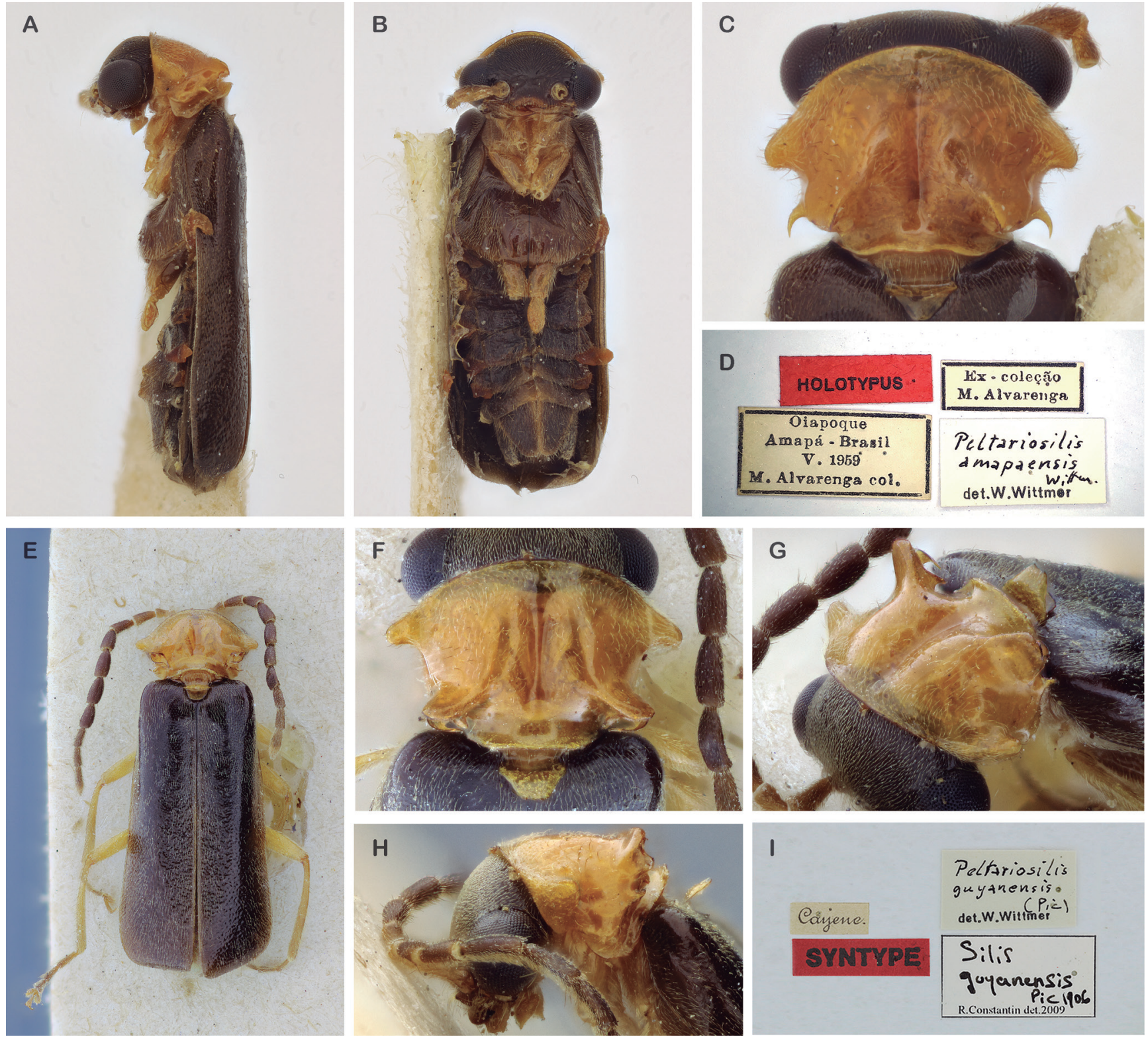

Figure 8. Primary types of Peltariosilis species. (A-D) Holotype of Silis (Peltariosilis) amapaensis Wittmer, 1966. (A) Lateral. (B) Ventral. (C) Pronotum. (D) Labels. (E-I) Lectotype of Silis guyanensis Pic, 1906. (E) Dorsal. (F-H) Pronotum. (I) Labels. 
postero-dorsally, distal margin dull, forming an inner obtuse peak and acute lateral peaks barely reaching sides of pronotum. Scutellum (Fig. 12I) with a long Y-shaped forked projection anteriorly; scutellar projection strongly narrowed at middle, elongate and broadly widened, forming apical fork with divergent apices; apices of fork acute, pointing latero-anteriorly. Elytra nearly parallel, apex rather truncate. Abdominal tergite VIII (Fig. 13F) subquadrate, nearly as long as wide, lateral margins sinuous, wider medially; glandular pores slightly protruding; distal margin with a shallow rounded median notch. Ventrite VII (Fig. 14F) wide, lateral margins sinuous, wider anteriorly and much narrower posteriorly, apex narrowly rounded near middle; internal margins nearly straight, divergent, halves broadly separated. Aedeagus (Fig. 16I-L): ventral plaque of tegmen lateroventral, elongated, nearly as long as internal sac, shorter than sclerites, flattened laterally, straight, apex sickle like, broadly curved dor- sally; parameres fused at base, parallel, broadly arched dorsally, apex acute; median lobe tubular, membranous, translucent; internal sac elongated, narrow, straight, weakly sclerotised; one single median dorsal sclerite, elongated, narrow, apex acute; paramedian dorsal sclerites robust, strongly sclerotised, elongated, straight with apex curved dorsally, rough, scratched; ventral sclerites slender, as long as internal sac, apex acute, slightly divergent; lateral sclerites indistinct or absent.

Female: General appearance and colouration similar to males, antennae thinner and shorter than in male, middle segments not thickened. Eyes smaller and less protruding. Pronotum as in (Fig. 11B).

Etymology: The specific epithet flavicornis is formed from the Latin words flavus (yellow) + cornis ("horn", antenna), referring to the yellow antennae.
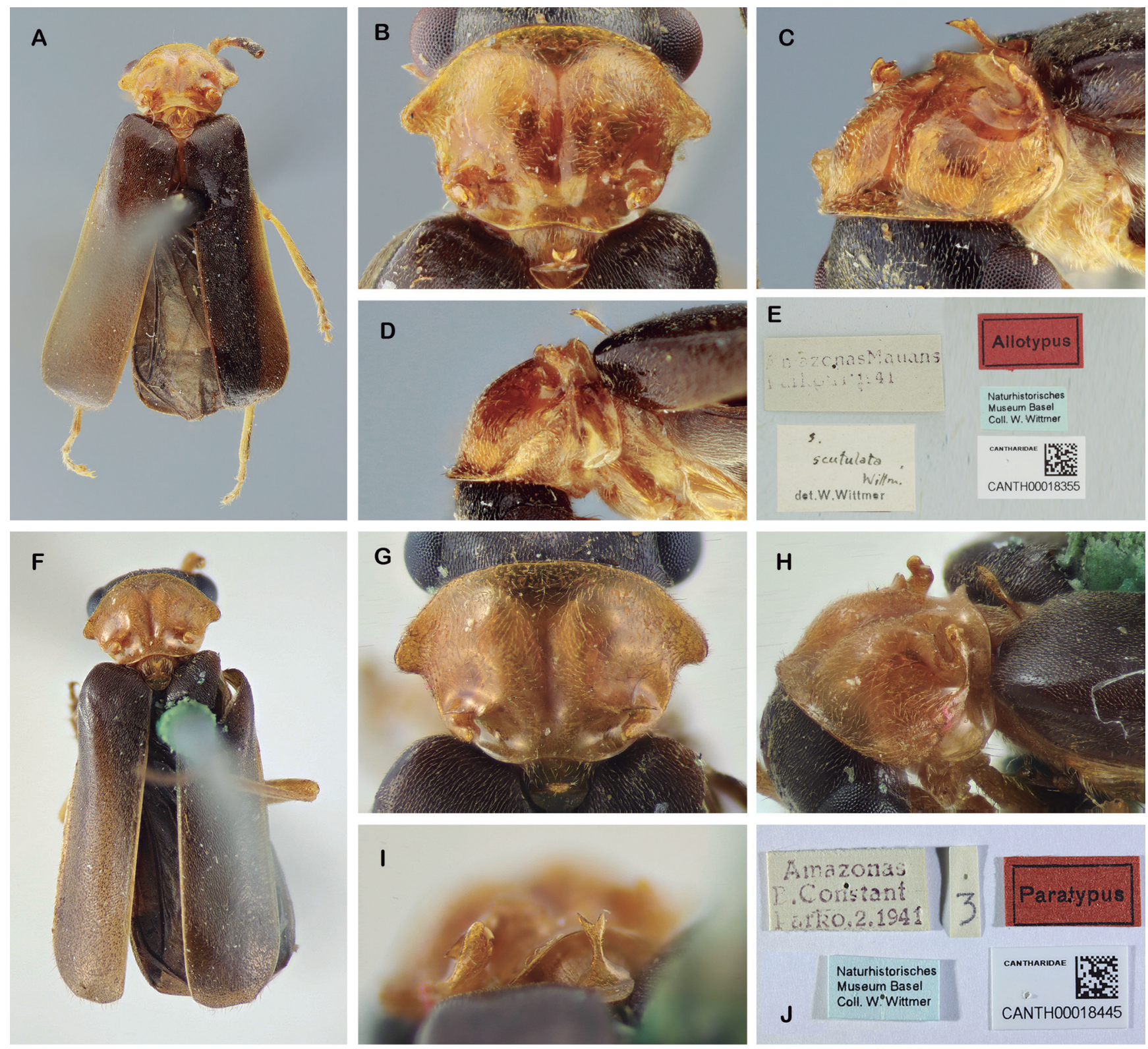

Figure 9. Types series of Peltariosilis scutulata (Wittmer, 1952) (A-E) Holotype ("allotype") of Silis (Peltariosilis) scutulata Wittmer, 1952. (A) Dorsal. (B-D) Pronotum. (E) Labels. (F-J) Paratype of Silis (Peltariosilis) scutulata Wittmer, 1952. (F) Dorsal. (G-I) Pronotum. (J) Labels. 
Distribution: Bolivia and Brazil (Amazonas and Mato Grosso states) (Fig. 18).

\section{Peltariosilis orientalis sp. nov. (Figs. 6D, 10J, 12J, 13G, 14G, 16M-P)}

\begin{abstract}
Type series: HOLOTYPE $\sigma^{\star}$ (CZMA): "Brasil(MA), C.N.Maranhão / REBIO -Res. Biol. Gurupi / $03^{\circ} 14^{\prime} 01^{\prime \prime} \mathrm{S} / 46^{\circ} 40^{\prime} 52^{\prime \prime} \mathrm{W} / /$ Armadilha Luminosa / 0307.v .2011, M. M. Abreu \& / J. A. Silva, cols" (Fig. 6D). PARATYPES (5 specimens): "Brasil(MA), C.N.Maranhão / REBIO -Res. Biol. Gurupi / 03 14'05"S/46 41'83"W // Arm. Luminosa Móvel / 07-15.I.2011, F. Limeira-de- / Oliveira \& M. M. Abreu, cols" (2 o", CZMA); "Brasil(MA), C.N.Maranhão / REBIO -Res. Biol. Gurupi / 03 14'05"S/46 41'83"W // Arm. Suspensa / 07-15. I .2011, F. Limeira-de- / Oliveira \& D. W. A. Marques" (1 o", CZMA); "Brasil (MA), Bom Jardim / REBIO-Res. Biol. Gurupi / Armad. Luminosa Base // 05 - 15. vi . 2010, J.C. / Silva, J. A. Silva, A. A. / Santos \& T. T. A. // MZUSP 31236" (1 0', MZUSP 31236); "Brasil(MA), C.N.Maranhão / REBIO -Res. Biol. Gurupi / $03^{\circ} 14^{\prime} 01^{\prime \prime} \mathrm{S} / 46^{\circ} 40^{\prime} 52^{\prime \prime} \mathrm{W} / /$ Armadilha Luminosa / 0307.v .2011, M. M. Abreu \& / J. A. Silva, cols // MZUSP 31237" (1 o', MZUSP 31237).
\end{abstract}

Diagnosis: Antennae not distinctly swollen, ventral surface slightly flattened, antennomeres I to basal half of III testaceous yellow, the following dark brown, X-XI orange yellow; pronotum and scutellum orange brown; elytra dark brown, testaceous yellow at lateral margins and along sutures; pronotum with low, dull dorsal projections, not reaching lateral margins of pronotum; wellmarked angles at laterobasal lobe; laterobasal lobes curved, apex rounded, divided in two flaps; scutellum with a long forked projection; apices short, acute, divergent, pointing latero-anteriorly. Peltariosilis orientalis sp. nov. is similar to $P$. scutulata by the general body colouration and shape of scutellar projection. Peltariosilis orientalis sp. nov. differs by the less prominent anterior margin of pronotum, continuous with frontolateral lobes, which are broader; dorsal projection of pronotum shallower, and shorter laterally; apices of scutellar projection shorter.

Description: Head black, except anterior margin of clypeus, dark brown; labium, maxillae, palpi and base of mandibles orange yellow, apex of mandibles dark brown; antennomeres I to basal half of III testaceous yellow, the following dark brown, $\mathrm{X}-\mathrm{XI}$ orange yellow; pronotum and scutellum orange brown, slightly translucent; elytra dark brown, testaceous yellow at lateral margins and along sutures; legs orange yellow, slightly infuscate at distal tarsomeres; thorax and abdomen dark brown.

Male: (Fig. 6D): Antennae rather short; antennomere I elongate, slightly wider apically, II short, slightly wider apically, III-IX nearly oblong, narrower proximally, flattened at ventral surface, X-XI slightly narrower, slender. Anterior margin of clypeus with a broad arcuate concave notch.
Pronotum (Fig. 10J): anterior margin broadly arched, continuous with frontolateral lobes; frontolateral lobe broad, sides rounded, sides behind frontolateral lobe rounded, slightly convergent; angles of laterobasal lobe obtuse, well-marked; laterobasal lobe directed posteriorly and dorsally, posterior margin straight, anterior margin sinuous, apex swollen, rounded, split, forming juxtaposed dorsal and ventral flaps; apex of ventral flap with a short membranous projection; dorsal projections low, transverse, directed postero-dorsally, distal margin slightly arcuate, forming an inner dull peak and short lateral peaks not reaching sides of pronotum. Scutellum (Fig. 12J) with a long forked projection anteriorly; scutellar projection strongly narrowed at middle and gradually widened and split towards apex, forming apical fork with arched sides; apices of fork short, divergent, pointing anteriorly. Elytra nearly parallel, apex rounded. Abdominal tergite VIII (Fig. 13G) subtrapezoidal, lateral margins broadly arched, wider anteriorly, slightly convergent posteriorly, glandular pores strongly protruding, distal margin with a broad and deep concave median notch. Ventrite VII (Fig. 14G) wide, trapezoidal, lateral margins wider anteriorly, convergent posteriorly; posterior margins straight laterally, with a low rounded projection internally. Aedeagus (Fig. 16M-P): ventral plaque of tegmen lateroventral, elongated, as long as internal sac, flattened laterally, curved internally, lateral margins arched, broad at base, narrowed at middle, wider at apex, apex broadly rounded with a dorsally curved acute tip; parameres fused at base and slightly divergent, regularly curved dorsally, apex narrowed with a small acute tip; median lobe membranous, translucent; internal sac elongated, narrow, straight, slightly sclerotised; one single median dorsal sclerite, elongated, narrow, apex acute; paramedian dorsal sclerites robust, strongly sclerotised, elongated, parallel, slightly curved dorsally, apex narrowed and divergent, pointing laterally; ventral sclerites slender, as long as internal sac, apex acute, slightly divergent; lateral sclerites indistinct or absent.

Female: Unknown.

Etymology: The specific epithet orientalis refers to the distribution of the species, in the easternmost reaches of Amazon forest.

Distribution: Brazil (Maranhão state) (Fig. 18).

\section{Peltariosilis cleidecostae sp. nov.} (Figs. 4A-D, 6E, 10K, 12K, 13H, 14H, 17A-D)

Type series: HOLOTYPE ơ (MZUSP 31239): “BRASIL. Acre, Mâncio Lima / Pé da Serra / 18.iii.2006. Armadilha de luz / AR Calor Col. // MZUSP 31239" (Fig.6E). PARATYPES: "Rio Branco / Acre Bras. / 29.X.1954 // Museum Frey / Tutzing // Naturhistorisches / Museum Basel / Coll. W. Wittmer" (1 $\left.\sigma^{x}, \mathrm{NHMB}\right)$.

Diagnosis: Antennae not distinctly swollen, ventral surface slightly flattened, antennomeres I-II and X-XI 
testaceous yellow, III-IX gradually infuscate; pronotum and scutellum testaceous yellow, slightly translucent; elytra dark brown, slightly lighter at lateral margins; pronotum with low, sharp dorsal projections, lateral peaks short, not reaching lateral margins of pronotum; apex of laterobasal lobes truncate, oblique, divided in two flaps; scutellum with a long forked projection strongly narrowed at middle and abruptly widened and split towards apex, forming apical fork, apices of fork acute, long, parallel, pointing anteriorly. Peltariosilis cleidecostae sp. nov. is very similar to $P$. brancuccii sp. nov. Both distinguish from other species by the shape of scutellar projection strongly narrowed at middle then abruptly widened, with parallel apices, and by the truncate and oblique apex of laterobasal lobes. Peltariosilis cleidecostae sp. nov. is distinguished by the more projected anterior margin of pronotum, dorsal projections wider, almost reaching lateral margins of pronotum, and shorter scutellar projection.

Description: Head black, except anterior margin of clypeus, light brown; labium, maxillae, palpi and base of mandibles pale yellow, apex of mandibles light brown; antennomeres I-II and X-XI testaceous yellow, III-IX gradually infuscate, dark brown; pronotum and scutellum testaceous yellow, slightly translucent; elytra dark brown, slightly lighter at lateral margins; legs testaceous yellow, slightly infuscate at tarsi; thorax and abdomen dark brown.

Male: (Fig. 6E): Antennae rather long; antennomere I elongate, slightly wider apically, || short, cylindrical, III-IX nearly oblong, narrower proximally, flattened at ventral surface, X-XI slightly narrower, slender. Anterior margin of clypeus with a broad arcuate concave notch. Pronotum (Fig. 10K): anterior margin arched, projected; frontolateral lobe narrow, not continuous with anterior margin, sides rounded; angles of laterobasal lobe indistinct, broadly rounded; laterobasal lobe directed posteriorly and dorsally, posterior margin rounded, apex truncate, oblique, split, forming juxtaposed dorsal and ventral flaps; apex of ventral flap with a broad membranous projection; dorsal projections low, transverse, directed postero-dorsally, distal margin arcuate, sharp, forming an inner acute peak and short lateral peaks not reaching sides of pronotum. Scutellum (Fig. 12K) with a long forked projection anteriorly; scutellar projection strongly narrowed at middle and abruptly widened and split towards apex, forming apical fork with arched sides; apices of fork acute, long, parallel, pointing anteriorly. Elytra nearly parallel, apex rounded. Abdominal tergite VIII (Fig. $13 \mathrm{H}$ ) nearly as long as wide, lateral margins broadly arched, wider medially, glandular pores not protruding, distal margin with a broad and deep concave median notch. Ventrite VII (Fig. 14H) wide, lateral margins arched, wider medially; posterior margins with broad rounded projections near middle. Aedeagus (Fig. 17A-D): ventral plaque of tegmen laterally, elongated, nearly as long as internal sac, flattened laterally, nearly straight, broader at base, gradually narrowing at apex and slightly curved internally, with a dorsally curved acute tip; parameres fused at base and slightly divergent, slightly curved dorsally, apex narrowed, strongly curved dorsally, with acute tip; median lobe membranous, translucent; internal sac elongated, narrow, straight; one single median dorsal sclerite, elongated, narrow, apex acute, arched at base, straight from middle; paramedian dorsal sclerites robust, strongly sclerotised, elongated, apex strongly curved dorsally, before the apex with a number of irregular furrows; ventral sclerites slender, shorter than internal sac, apex acute, slightly divergent; lateral sclerites indistinct or absent.

Female: Unknown.

Etymology: The specific epithet is patronymic, named for Professor Cleide Costa (MZUSP) on the occasion of her $80^{\text {th }}$ birthday, in honour of her contributions to the systematics of Coleoptera and pioneering studies on immature stages of Neotropical beetles.

Distribution: Brazil (Acre state) (Fig. 18).

Remarks: Further information on the type locality: "Igarapé do Amor, near IBAMA office. Going up Moa River, first right affluent" [ca. $7^{\circ} 28^{\prime} \mathrm{S}, 73^{\circ} 37^{\prime} \mathrm{W}$ ] (Adolfo Calor, personal communication).

\section{Peltariosilis brancuccii sp. nov. (Figs. 6F, 10L, 12L, 13I, 14I, 17E-H)}

Type series: HOLOTYPE o" (NHMB): "Cobija / Bolivia / 12. 1956 Prosen // 21370 // Naturhistorisches / Museum Basel / Coll. W. Wittmer". PARATYPE o" (CEMT): "Brasil:

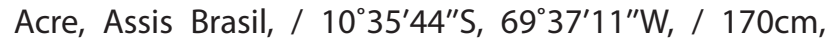
26.ix.2016, wild / environment, light trap / (CDC), Ortiz \& Borges. // Peltariosilis / n. sp. / near scutulata / det. M. Geiser 2018" (Fig. 6F).

Diagnosis: Antennae not distinctly swollen, ventral surface slightly flattened, antennomeres I-II light brown, III-IX dark brown to black, X-XI orange brown; pronotum and scutellum orange brown to light brown, slightly translucent; elytra dark brown to black, slightly lighter at lateral margins; pronotum with low, sharp dorsal projections, lateral peaks very short, not reaching lateral margins of pronotum; apex of laterobasal lobes truncate, oblique, divided in two flaps; scutellum with a long forked projection strongly narrowed at middle and abruptly widened and split towards apex, forming apical fork, apices of fork acute, long, parallel, pointing anteriorly. Peltariosilis brancuccii sp. nov. is very similar to $P$. cleidecostae sp. nov. It distinguishes from the later by the less projected anterior margin of pronotum, contiguous with frontolateral lobes, dorsal projections narrower, and longer scutellar projection.

Description: Head black; labium, maxillae, palpi and base of mandibles brown, apex of mandibles dark 
brown; antennomeres I-II light brown, III-IX dark brown to black, X-XI orange brown; pronotum and scutellum orange brown to light brown, slightly translucent; elytra dark brown to black, slightly lighter at lateral margins; legs orange brown, infuscate at tarsi; thorax and abdomen dark brown.
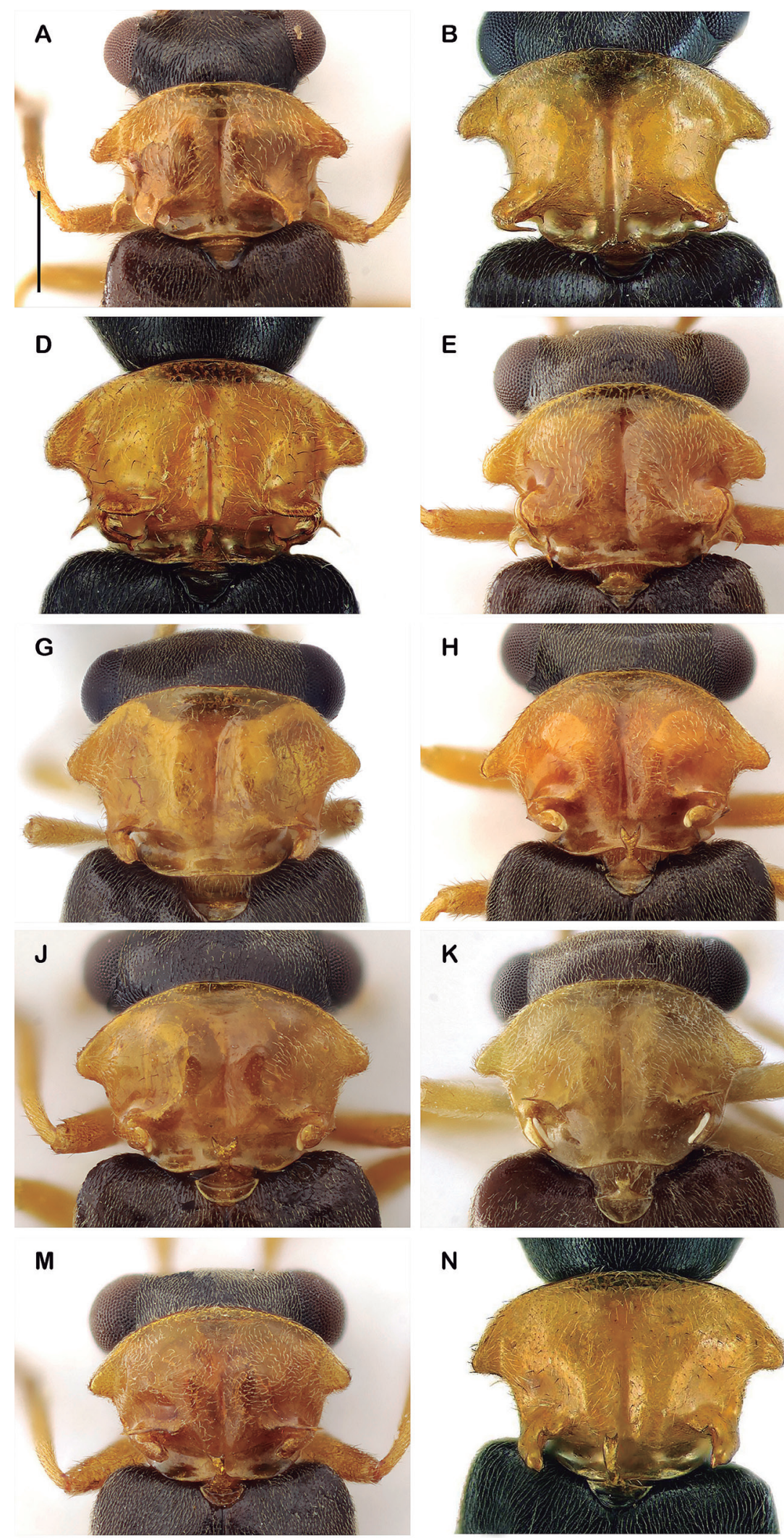
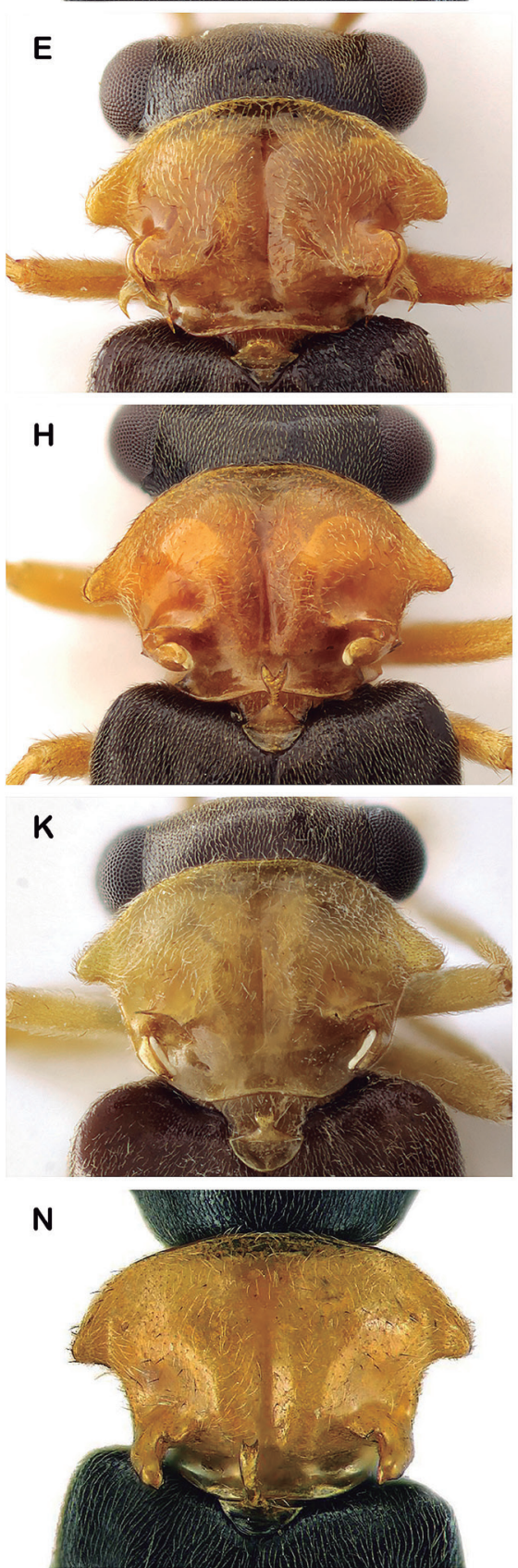
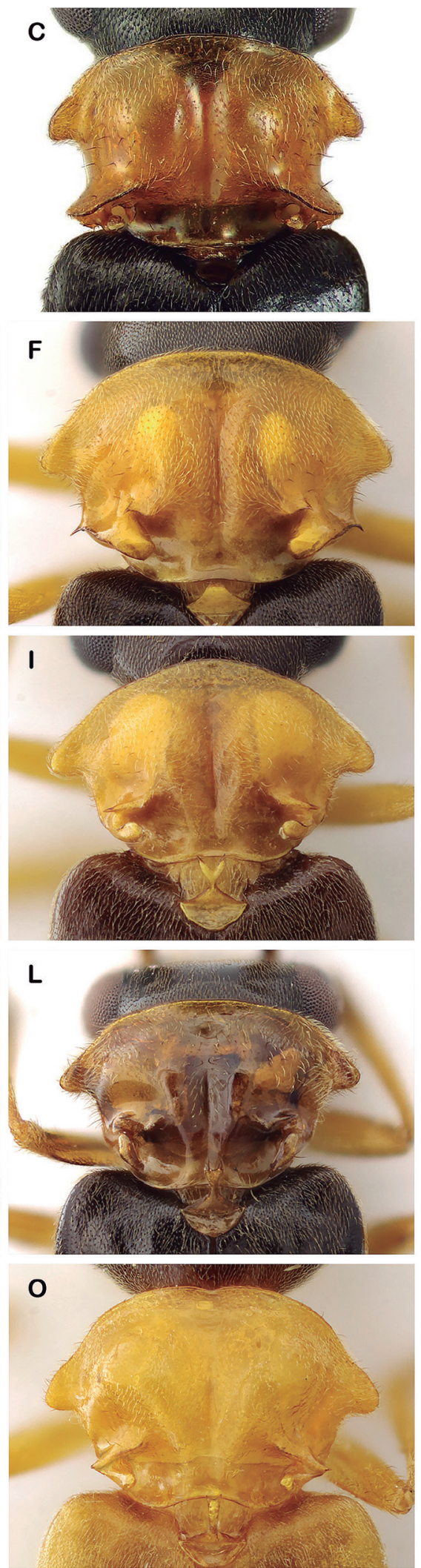

Figure 10. Pronotum of Peltariosilis species, males. (A) P. amapaensis (Wittmer, 1966). (B) P. guyanensis (Pic, 1906). (C) P. mensaemontis Constantin, 2017. (D) P. lamellata Constantin, 2017. (E) P. diversicollis sp. nov. (F) P. major sp. nov. (G) P. gracilicornis sp. nov. (H) P. scutulata (Wittmer, 1952). (I) P. flavicornis sp. nov. (J) P. orientalis sp. nov. (K) P. cleidecostae sp. nov. (L) P. brancuccii sp. nov. (M) P. parviscutellaris sp. nov. (N) P. mitarakaemontis Constantin, 2017. (0) P. brunneoapicalis sp. nov. B, C, D and N modified from Constantin (2017). All figures in the same scale, except B, C, D and N. Scale bar $=0.5 \mathrm{~mm}$. 

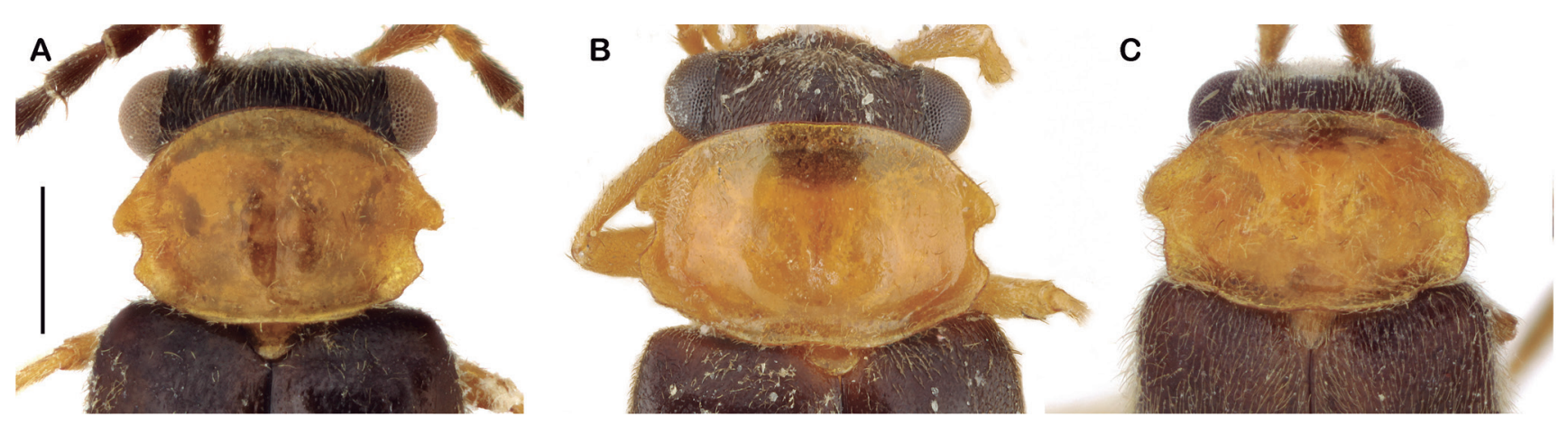

Figure 11. Pronotum of Peltariosilis species, females. (A) P. amapaensis (Wittmer, 1966). (B) P. flavicornis sp. nov. (C) P. parviscutellaris sp. nov. All figures in the same scale. Scale bar $=0.5 \mathrm{~mm}$.

Male: (Fig. 6F): Antennae rather long; antennomere I elongate, slightly wider apically, II short, cylindrical, slightly wider apically, III-IX nearly oblong, narrower proximally, flattened at ventral surface, X-XI slightly narrower, slender. Anterior margin of clypeus with a broad arcuate concave notch. Pronotum (Fig. 10L): anterior margin arched, slightly projected; frontolateral lobe narrow, continuous with anterior margin, sides rounded; angles of laterobasal lobe broadly rounded, barely distinct; laterobasal lobe directed posteriorly
A

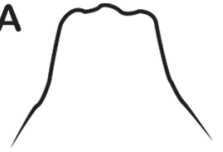

D

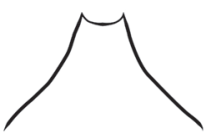

G<smiles>C1CCCCCCC1</smiles>

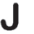

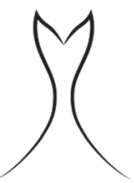

M

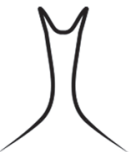

B

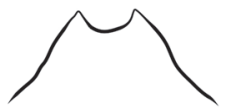

E

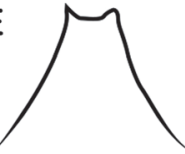

H

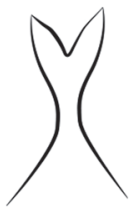

K

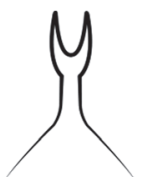

N

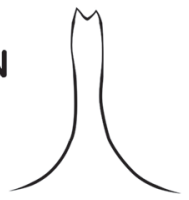

C
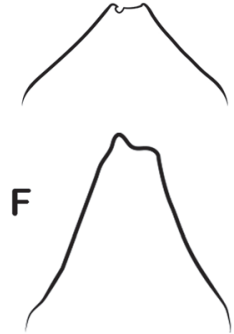

I

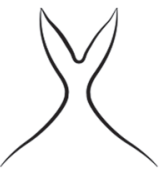

L
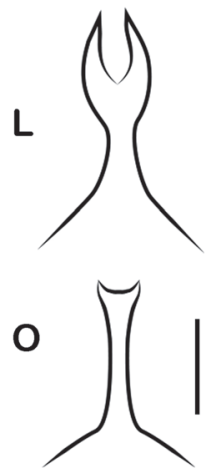

Figure 12. Scutellar projection of Peltariosilis species, males. (A) P. amapaensis (Wittmer, 1966). (B) P. guyanensis (Pic, 1906). (C) P. mensaemontis Constantin, 2017. (D) P. lamellata Constantin, 2017. (E) P. diversicollis sp. nov. (F) P. major sp. nov. (G) P. gracilicornis sp. nov. (H) P. scutulata (Wittmer, 1952). (I) P. flavicornis sp. nov. (J) P. orientalis sp. nov. (K) P. cleidecostae sp. nov. (L) P. brancuccii sp. nov. (M) P. parviscutellaris sp. nov. (N) P. mitarakaemontis Constantin, 2017. (0) P. brunneoapicalis sp. nov. All figures in the same scale. Scale bar $=0.5 \mathrm{~mm}$ and dorsally, posterior margin rounded, apex truncate, oblique, split, forming juxtaposed dorsal and ventral flaps; apex of ventral flap with a broad membranous projection; dorsal projections low, transverse, directed postero-dorsally, distal margin arcuate, sharp, forming an inner acute peak and very short lateral peaks not reaching sides of pronotum. Scutellum (Fig. 12L) with a long, forked projection anteriorly; scutellar projection strongly narrowed at middle and abruptly widened and split towards apex, forming apical fork with arched sides; apices of fork acute, long, parallel, pointing anteriorly. Elytra nearly parallel, apex rounded. Abdominal tergite VIII (Fig. 13I) subquadrate, lateral margins broadly arched, wider medially, glandular pores protruding, distal margin sinuous, with a shallow and narrow concave median notch. Ventrite VII (Fig. 14I) wide, lateral margins arched, wider medially, narrowed posteriorly; posterior margin slightly arched. Aedeagus (Fig. 17E-H): ventral plaque of tegmen laterally elongated, slightly shorter than internal sac, flattened laterally, slightly convergent at base, parallel from middle, strongly narrowed at apex, with a sharp acute tip curved dorsally; parameres fused at base and nearly parallel, slightly curved dorsally, apex rounded, with small acute tip; median lobe membranous, translucent; internal sac elongated, narrow, straight; one single median dorsal sclerite, elongated, narrow, apex acute, arched at base, straight from middle; paramedian dorsal sclerites robust, strongly sclerotised, elongated, apex broadly arched, curved dorsally, before the apex with several irregular furrows of variable length; ventral sclerites slender, shorter than internal sac, apex acute, slightly divergent, slightly divergent; lateral sclerites indistinct or absent.

Female: Unknown.

Etymology: The specific epithet is patronymic, named in honour of the second author's late advisor, Dr Michel Brancucci (1950-2012), in appreciation of his contributions to Cantharidae systematics and in gratitude for his constant encouragement to specialise in the neglected "malacoderm" families Cantharidae and Prionoceridae.

Distribution: Bolivia and Brazil (Acre state) (Fig. 18). 


\section{Peltariosilis parviscutellaris sp. nov. (Figs. 1B, 7A, 10M, 11C, 12M, 13J, 14J, 17I-L)}

Type series: HOLOTYPE ơ (MPEG):"Icoraci / 19.12.1961 // Brasil, PA / J. \& B. Bechyné" (Fig. 7A). PARATYPES (16 specimens): "Icoraci / 19.12.1961 // Brasil, PA / J. \& B. Bechyné" (3 o", MPEG, 3 o", MZUSP 31244-31246); "Icoraci, Belém / PA, Brasil / 3.VIII.1962 / J. Bechyné col." (3 ơ, MZUSP 31247-31249); "Marituba / 7.11.1961 // Brasil, PA / J. \& B. Bechyné" (1 ơ, MPEG);"Benevides / 20.11.1961 // Brasil, PA / J. \& B. Bechyné" (1 ơ, MPEG); “Utinga / 7.12.1961 // Brasil, PA / J. \& B. Bechyné" (1 ơ, MPEG); “Utinga / 30.12.1960 // Brasil, PA / J. \& B. Bechyné" (1 \&, MPEG); "Belém, Utinga / 24.III.1977 // Brasil Pará / M F Torres" (2 \&, MZUSP 3125031251); “Benfica / 31.5.1961 // Brasil, PA / J. \& B. Bechyné” (1, MPEG).

Diagnosis: Antennae slightly swollen at middle antennomeres, ventral surface slightly flattened; antennomeres I-II testaceous brown, III-IX light to dark brown. X-XI orange brown; pronotum and scutellum orange brown, slightly translucent; elytra dark brown, lighter at lateral margins and along suture; apex of laterobasal lobe of pronotum rounded, split, forming juxtaposed dorsal and ventral flaps; dorsal projections well-projected, transverse, distal margin arcuate, sharp, very wide and acute lateral peaks surpassing sides of pronotum; scutellum with very slender lamellar projection, narrower near base and slightly wider distally, apices slightly projected, not forming well-defined fork. Peltariosilis parviscutellaris
A

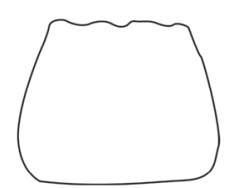

D

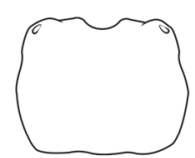

G

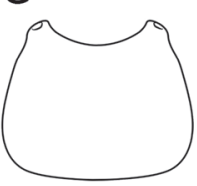

J

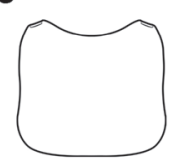

B

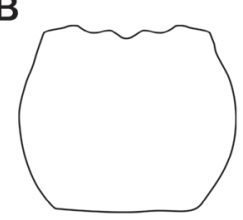

E

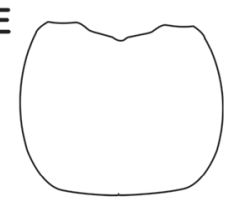

H
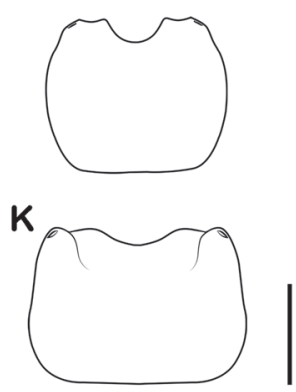

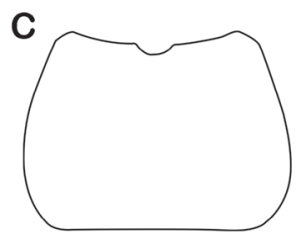

$\mathbf{F}$

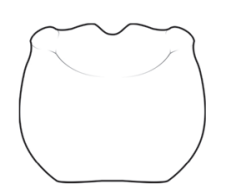

I

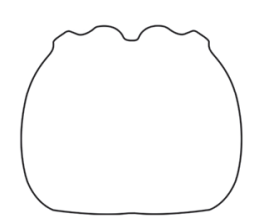

A

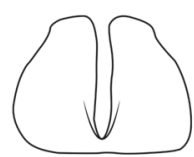

D

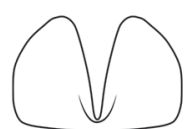

G

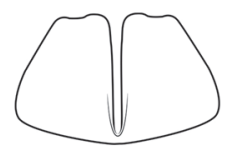

J

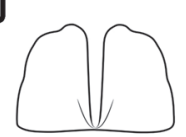

B

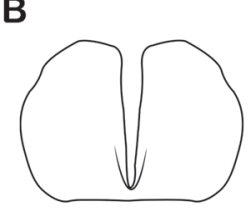

E

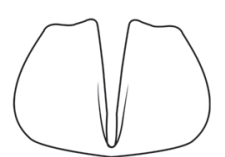

H

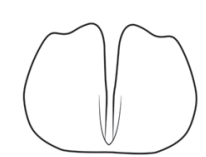

$\mathrm{K}$

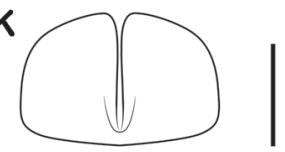

C

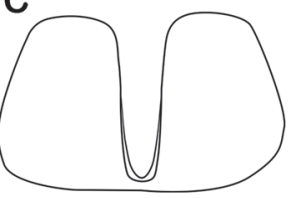

$\mathbf{F}$

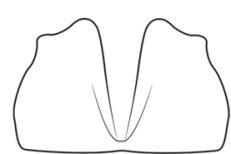

1

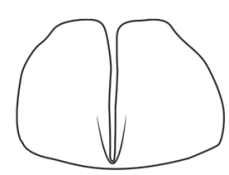

Figure 14. Ventrite VII of Peltariosilis species, males. (A) P. amapaensis (Wittmer, 1966). (B) P. diversicollis sp. nov. (C) P. major sp. nov. (D) P. gracilicornis sp. nov. (E) P. scutulata (Wittmer, 1952). (F) P. flavicornis sp. nov. (G) P. orientalis sp. nov. (H) P. cleidecostae sp. nov. (I) P. brancuccii sp. nov. (J) P. parviscutellaris sp. nov. (K) P. brunneoapicalis sp. nov. All figures in the same scale. Scale bar $=0.5 \mathrm{~mm}$.
Figure 13. Tergite VIII of Peltariosilis species, males. (A) P. amapaensis (Wittmer, 1966). (B) P. diversicollis sp. nov. (C) P. major sp. nov. (D) P. gracilicornis sp. nov. (E) P. scutulata (Wittmer, 1952). (F) P. flavicornis sp. nov. (G) P. orientalis sp. nov. (H) P. cleidecostae sp. nov. (I) P. brancuccii sp. nov. (J) P. parviscutellaris sp. nov. (K) P. brunneoapicalis sp. nov. All figures in the same scale. Scale bar $=0.5 \mathrm{~mm}$. sp. nov. is similar to P. mitarakaemontis and P. brunneoapicalis sp. nov. by the shape of scutellar projection not

Description: Head black, except anterior margin of clypeus, light brown; mandibles, labium, maxillae and palpi light brown; antennomeres I-II testaceous brown, III-IX brown, lighter at lateral margins and along suture; legs orange brown, infuscate at apex of tibiae and tarsi; tho-

Male: (Fig. 7A): Antennae rather long; antennomere I elongate, slightly wider apically, || short, cylindrical, slightly wider apically, III-IX nearly oblong, narrower proximally, flattened at ventral surface, X-XI slightly narrower, slender. Anterior margin of clypeus with a broad arcuate concave notch. Pronotum (Fig. 10M): anterior broad, continuous with anterior margin, sides rounded; angles of laterobasal lobe broadly rounded, indistinct; laterobasal lobe directed posteriorly and dorsally, apex rounded, split, forming juxtaposed dorsal and ventral ; dorsal projections well-projected, transverse, diforming an inner dull peak and very wide and acute lateral peaks surpassing sides of pronotum. Scutellum forming distinct fork. It differs from these species by the sharp, broad and transversal dorsal projections of ght to dark brown. X-XI orange brown; pronotum and 
(Fig. 12M) with very slender lamellar projection, narrower near base and slightly wider distally, apices acute, slightly projected, not forming well-defined fork. Elytra nearly parallel, apex rounded. Abdominal tergite VIII (Fig. 13J) quadrate, lateral margins nearly parallel, glandular pores not protruding; posterior margin broadly concave. Ventrite VII (Fig. 14J) small, lateral margins sinuate, wider anteriorly, with a small constriction at middle; posterior margin with a small rounded projection near middle. Aedeagus (Figs. 17I-L): ventral plaque of tegmen lateroventrally, elongated, as long as internal sac, flattened laterally, curved internally at middle and divergent at apex, margins sinuated, narrower at middle, wider at apex, apex broadly rounded with a dorsally curved acute tip; parameres fused at base and nearly parallel, slightly divergent, regularly and strongly curved dorsally, apex rounded with a small acute tip; median lobe membranous, translucent; internal sac elongated, narrow, straight, nearly as long as ventral, median and paramedian dorsal sclerites; median dorsal sclerite indistinct or absent; paramedian dorsal sclerites slender, strongly sclerotised, elongated, parallel, nearly straight, slightly curved dorsally, apex abruptly narrowed, sharp, pointing dorsally; ventral sclerites slender, as long as internal sac, apex acute, slightly divergent; lateral sclerites indistinct or absent.

Female: General appearance and colouration similar to males; antennae shorter, antennomeres slightly conical, more densely pubescent, middle antennomeres not swollen; clypeus (Fig. 1B) projected anteriorly in a conical beak; pronotum (Fig. 11C) rather transverse, wider than long, frontolateral lobes very broad and short; laterobasal lobe short, slightly projecting, dorsal projections absent; median and basal grooves shallow; scutellum simple, without projection; ventrite VII with a deep and broad median notch on distal margin, entire, not divided in two halves.

Etymology: The specific epithet parviscutellaris is formed from the Latin word parvus (small, weak) + scutellum, referring to the small size of scutellar fork in comparison with closely related species.

Distribution: Brazil (Pará state) (Fig. 18).

\section{Peltariosilis mitarakaemontis Constantin, 2017 (Figs. 7B, 10N, 12N)}

\section{Peltariosilis mitarakaemontis Constantin, 2017: 63.}

Type material examined: PARATYPE o" (CCo): "Guyane fr., massif Mitaraka / camp DZ, light trap / 26.II. 2015, alt. $310 \mathrm{~m} \mathrm{/} 2^{\circ} 14^{\prime} 4^{\prime \prime} \mathrm{N}-54^{\circ} 27^{\prime} 2^{\prime \prime} \mathrm{W} / \mathrm{J}$.Touroult,E. Poirier,J.H.Yvinec // La Planète Revisitée-MNHN / PNI Guyane 2015-APA-973-1 /collecte, tri \& préparation / Société entomologique Antilles-Guyane // PARATYPE / Peltariosilis / mitarakaemontis / Constantin i.l. / R. Constantin des. 2016".
Diagnosis: Head black, antennae brown, except antennomeres I-III and X light brown, $\mathrm{XI}$ yellow, pronotum and scutellum orangish, elytra dark brown, legs yellow. Male (Fig. 7B): Antennae long, antennomeres IV-XI slender, not swollen, slightly wider at apex. Pronotum (Fig. 10N): anterior margin broadly arched; frontolateral lobes small, not strongly projected; sides behind frontolateral lobe nearly straight, slightly convergent; laterobasal lobe robust, projected dorsally, not strongly curved, dorsal flap broad, apex rounded; dorsal projections very small, forming a weak angulate protuberance meeting laterobasal lobe laterally. Scutellum (Fig. 12N) with very slender lamellar projection, narrower near base and slightly wider distally, apices slightly projected, not forming well-defined fork. Peltariosilis mitarakaemontis is similar to P. parviscutellaris sp. nov. and $P$. brunneoapicalis sp. nov. by the shape of scutellar projection not forming distinct fork. It differs from these species by the uniform dark brown colouration of elytra, and by pronotum with very small dorsal projection and laterobasal lobes not strongly curved.

Distribution: French Guiana (Fig. 18).

\section{Peltariosilis brunneoapicalis sp. nov. (Figs. 7C, 100, 120, 13K, 14K, 17M-P)}

Type series: HOLOTYPE $\sigma^{\prime \prime}$ (INPA): "BRASIL. Amazonas, Manaus / ZF - 2, km 34, Base LBA / 02 35'37"S, 60'12'39"W / 09-11.vii.2008. Arm. luz no chão / JA Rafael \& FF Xavier Fo. Col." (Fig. 7C). PARATYPES (3 specimens): "BRASIL. Amazonas, Manaus / Parque das Laranjeiras / 15-30.i.1981 / CDC light trap. Eq. J. Arias Col." (1 o', INPA); "Brasil, Amazonas / Reserva Ducke / 26 km NE Manaus / Barbosa, M. G. V. // Plot C / Malaise 5 / Nov/95 // MZSP 31240" (1 o", MZSP 31240); "BRASIL: AM / R. Duke / 30-IV-1982 / J. A. Rafael // MZSP 31241" (1 o", MZSP 31241); "BRAZIL: Am. / Reserva Ducke / 26km NE. Manaus / Barbosa, M.G.V. // Plot C / FIT 3 / March 1995 // 68.1 // BMNH\{E\} / 2003-84" (1 ơ BMNH).

Diagnosis: Antennae testaceous yellow on antennomeres I-III and IX-XI, light to dark brown on IV-VIII, pronotum and scutellum orange yellow, elytra mostly testaceous yellow, with light to dark brown irregular patches at apex. Pronotum with frontolateral lobes long and straight, projected posteriorly and dorsally; angles of frontolateral lobe indistinct; dorsal projections sharp, diagonal, with lateral peaks meeting base of laterobasal lobes. Scutellum with very slender lamellar projection, narrower near base and slightly wider distally, apices slightly projected, not forming well-defined fork. Peltariosilis brunneoapicalis sp. nov. differs from all other species by the elytra mostly testaceous yellow with irregular brown patches.

Description: Head black, except anterior margin of clypeus, orange brown; base of mandibles, labium, maxillae and palpi testaceous yellow; antennae testaceous yellow in antennomeres I-III and IX-XI, antennomeres 
IV-VIII light to dark brown; thorax and abdomen light to dark brown; pronotum and scutellum orange yellow, slightly translucent; legs and elytra testaceous yellow, except for broad, irregular light to dark brown apical elytral patches.

Male: (Fig. 7C): Antennae long; antennomere I elongate, slightly wider apically, II short, slightly wider apically, III-X nearly cylindrical, narrower proximally, flat at ventral surface and globose at dorsal surface, X-XI narrower, slender, filiform. Anterior margin of clypeus with a broad arcuate concave notch. Pronotum (Fig. 100): anterior margin strongly arched; frontolateral lobe distinct, broad, projected laterally, arched anteriorly and straight posteriorly; sides behind frontolateral lobe rounded, with indistinct angles of laterobasal lobe; laterobasal lobes long, straight, directed posteriorly and dorsally, apex rounded, not swollen, dorsal and ventral flaps juxtaposed, barely distinct; dorsal projections razor blade like, straight, diagonal, directed postero-dorsally, distal margin sharp, forming an inner obtuse peak and acute lateral peaks meeting base of laterobasal lobes. Scutellum (Fig. 120) with very slender lamellar projection, narrower near base and slightly wider distally, apices acute, slightly projected, not forming well-defined fork. Elytra nearly parallel, apparently wider distally, apex rounded. Abdominal tergite VIII (Fig. 13K) subrectangular, wider than long, lateral margins slightly arched, distal margin with a broadly arched median notch; ventrite VII (Fig. 14K) wide, lateral and distal margins broadly arched, longer at middle. Aedeagus (Fig. 17M-P): ventral plaque of tegmen lateroventral, elongated, straight, very broad, flattened laterally, margins strongly sinuous, apex broadly rounded with a dorsally curved

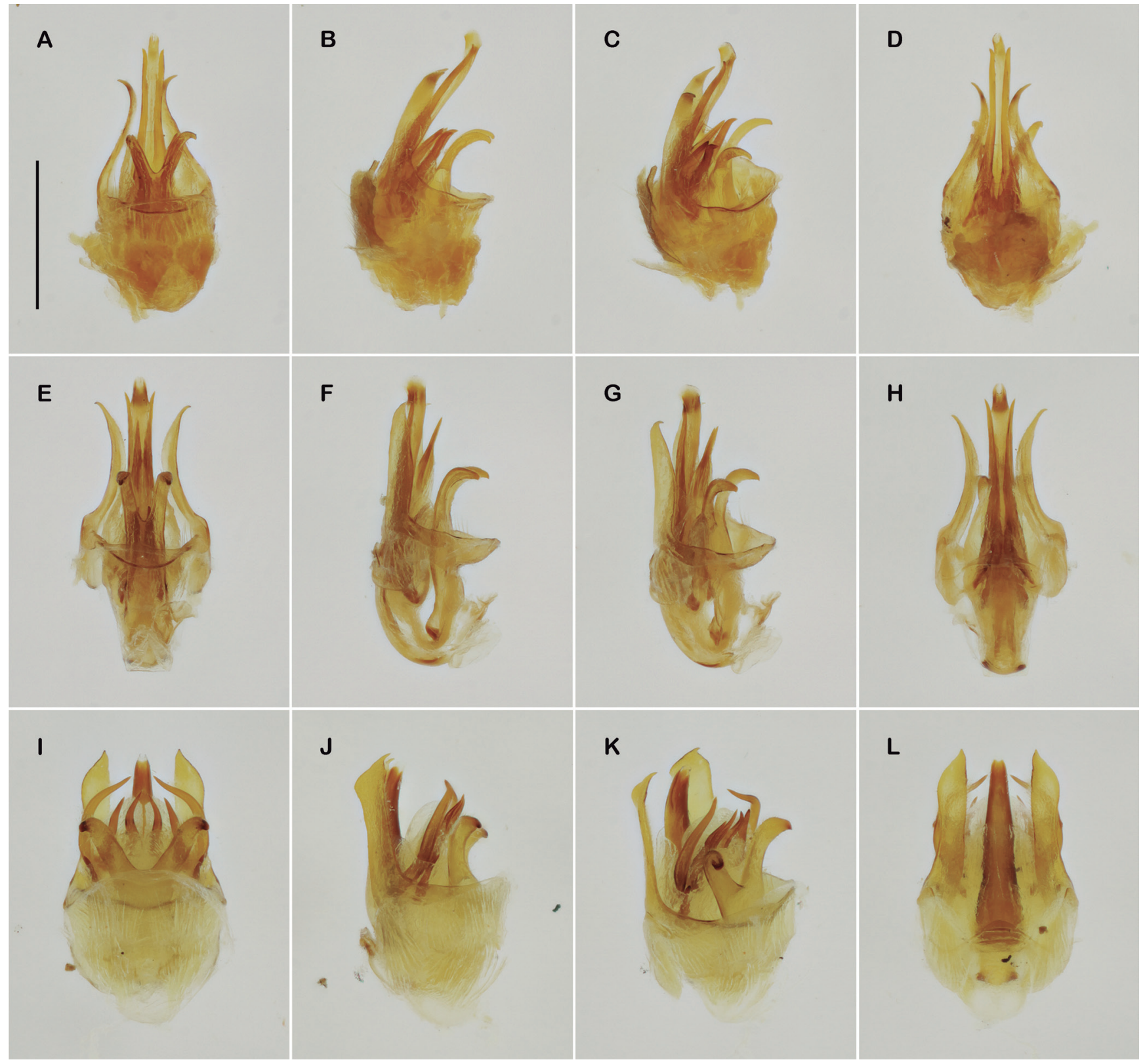

Figure 15. Aedeagi of Peltariosilis species (dorsal, lateral, dorsolateral and ventral views). (A-D) P. amapaensis (Wittmer, 1966). (E-H) P. diversicollis sp. nov. (I-L) P. major sp. nov. All figures in the same scale. Scale bar $=0.5 \mathrm{~mm}$. 
sharp tip; parameres fused at base and strongly divergent, flattened, narrower at base, widening towards apex, regularly curved dorsally, apex obtuse; median lobe membranous, translucent; internal sac elongated, narrow, slightly curved ventrally at apex, much longer than sclerites; median dorsal sclerites elongated, fused at base, split at apex, acute; paramedian dorsal sclerites very short, concealed behind parameres, strongly scle-

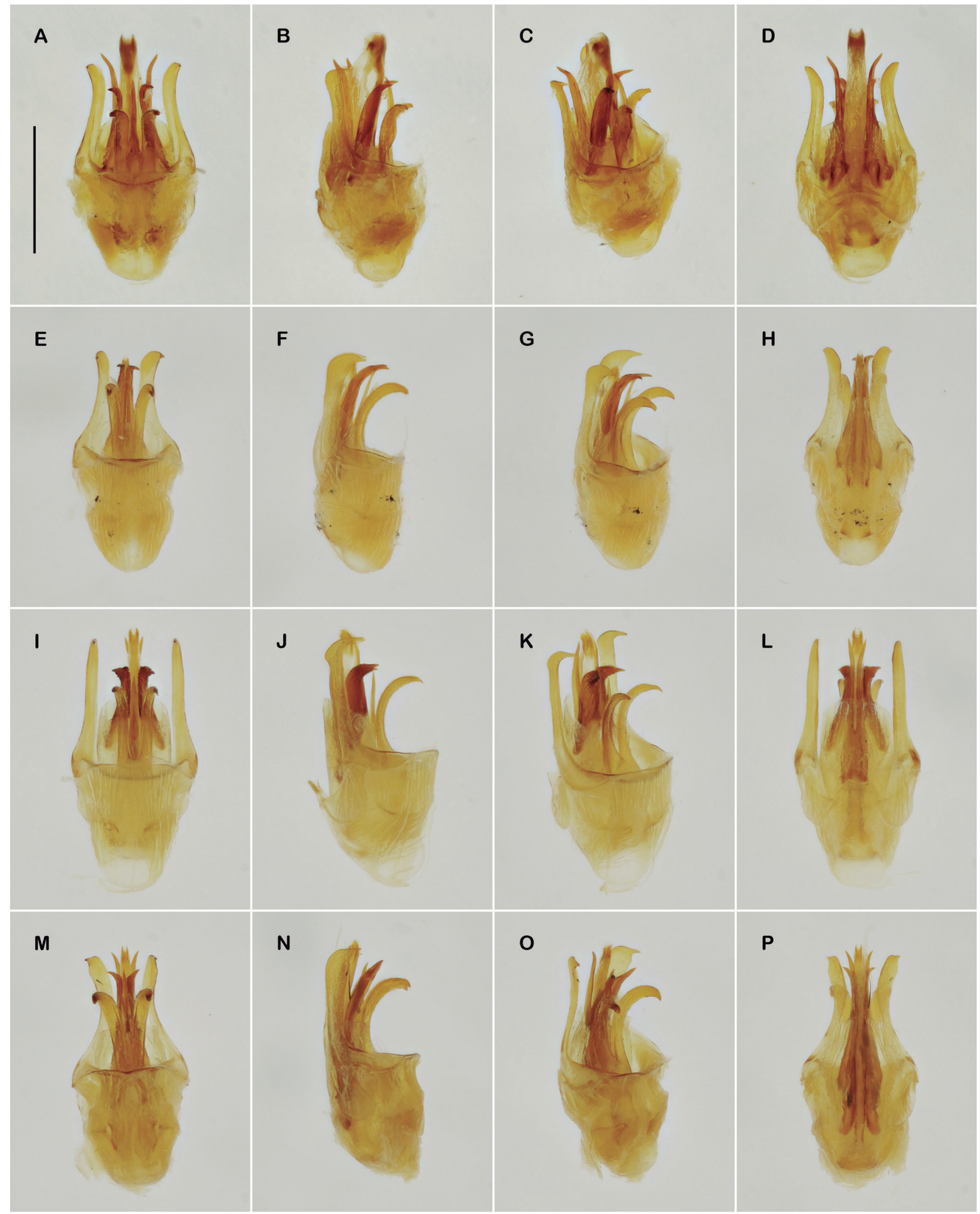

Figure 16. Aedeagi of Peltariosilis species (dorsal, lateral, dorsolateral and ventral views). (A-D) P. gracilicornis sp. nov. (E-H) P. scutulata (Wittmer, 1952). (I-L) P. flavicornis sp. nov. (M-P) P. orientalis sp. nov. All figures in the same scale. Scale bar $=0.5 \mathrm{~mm}$. 
rotised, acute, curved dorsally; ventral sclerites slender, acute, strongly curved dorsally, convergent; lateral sclerites indistinct or absent.

Female: Unknown.

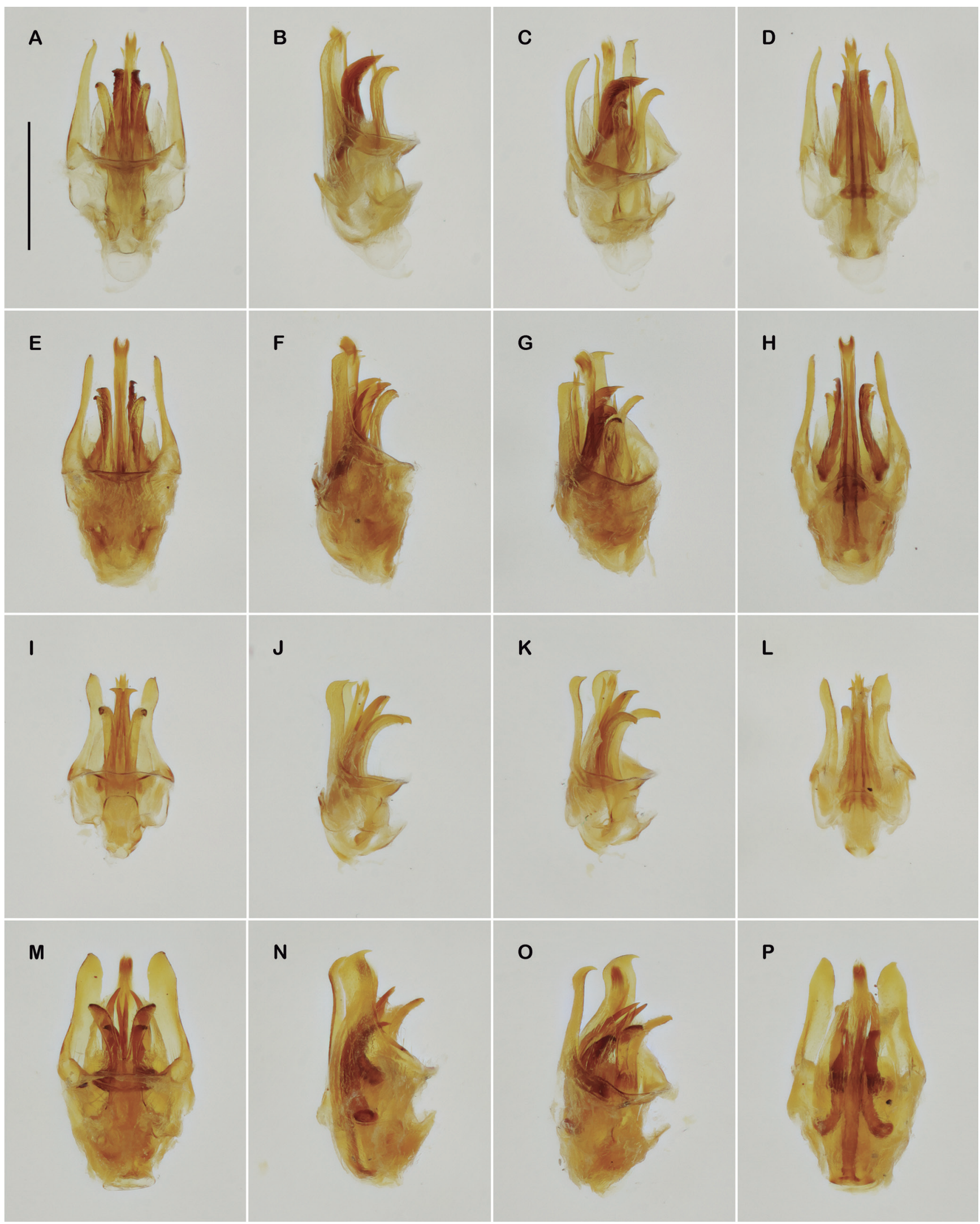

Etymology: The specific epithet brunneoapicalis is formed from the Latin words brunneus (brown) + apicalis (apical), referring to the elytral apical brown spots.

Distribution: Brazil (Amazonas state) (Fig. 18). 


\section{Distribution and natural history of Peltariosilis}

With the new species described herein, the number of known Peltariosilis species is more than doubled. In addition to the 15 species now recognised, we have currently unidentified material from some more localities (see Fig. 18) currently pending further studies, so we can expect the number of species to rise even further as more material becomes available. All of the species are restricted to the Amazonian subregion of the Neotropical region (sensu Morrone, 2000), with only P. flavicornis extending into the southern fringes of that area in Mato Grosso state, Brazil.

Based on the limited biological data available to us, mostly just the labels of the specimens, we can extrapolate that Peltariosilis are generally associated with forests, and most often with humid evergreen forests. One species, $P$. orientalis from Maranhão, was collected in an ecotone area between Amazon forest, savannah and Atlantic rainforest, with the exact habitat being unknown. Peltariosilis lamellata was collected at a light trap placed in a rocky savannah on top of a table mountain. Peltariosilis scutulata and P. cleidecostae are recorded from flooded forests (igarapés) in Acre and Roraima states. Most species were collected at rather low elevations, with only P. mensaemontis reaching $800 \mathrm{~m}$ on one of the "inselbergs" in French Guiana.

An interesting result of our study is the high morphological similarity between some of our species (e.g., P. cleidecostae sp. nov. and P. brancuccii sp. nov.), which makes them hard to separate based on external characters and has already led to some confusion regarding Wittmer's definition of $P$. scutulata. Despite the highly complex and diverse pronotum and scutellum characters in the male, it is recommended to always examine the aedeagus for reliable identification, with the females being only identifiable when unambiguously associated with a male at the same locality. All this indicates that Peltariosilis have radiated within the Amazonian subregion rather recently. The once widespread hypothesis of allopatric speciation in Amazonian taxa due to Pleistocene forest fragmentation (e.g., Vanzolini \&Williams, 1970) is now increasingly being challenged (e.g., Knapp \& Mallet, 2003; Bush \& Oliveira, 2006). As long as the real biogeographic history of the Amazon forests is very much disputed (Hooghiemstra, 2001), we would like to refrain from making any guesses on when Peltariosilis could have radiated. The complex structures of pronotum and scutellum in male Peltariosilis, likely associated with sexual pheromones (Eberhard, 2006), may have been driving speciation by increasing the reproductive isolation between populations.

It is evident that some Peltariosilis species have a rather restricted distribution within the Amazonian subregion. A number of species (P. gracilicornis, P. lamellata, P. major, P. mensaemontis, P. mitarakaemontis, P. orientalis) are only known from a single locality. The species known from multiple localities are confined to either South or North of the Amazon main course, with several species ( $P$. amapaensis, $P$. diversicollis, $P$. guyanensis, P. lamellata, $P$. major, $P$. mensaemontis, $P$. mitarakaemontis) restricted to the Guianas including Amapá ("Moist Guianian province" of Morrone, 2000).

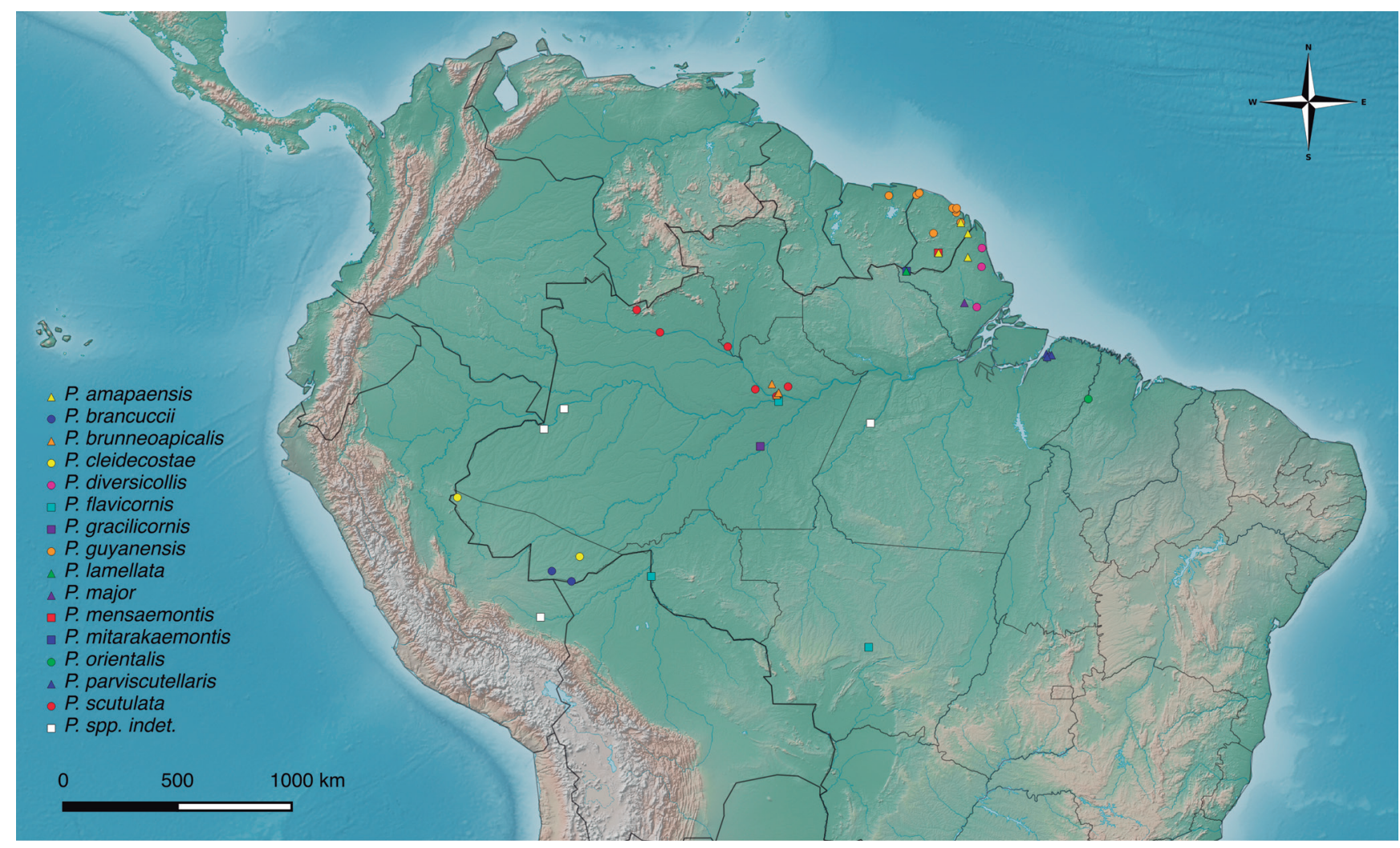

Figure 18. Distribution of Peltariosilis species. 
The vast majority of the specimens examined herein came from light traps. Two species ( $P$. major and $P$. scutulata) were reportedly beaten from vegetation during the day. Peltariosilis amapaensis was collected in flight intercept traps and P. mitarakaemontis was present in SLAM (sea-land-aerial-Malaise trap), as well as light trap samples (Constantin, 2017). Based on this, we can assume that Peltariosilis are predominantly nocturnal. The collecting dates of most type series indicate that many species may also be highly seasonal, with a short adult lifespan, although further material is needed to confirm this assumption.

\section{ACKNOWLEDGEMENTS}

We would like to thank the following curators for providing loans and access to specimens for this study: Augusto Henriques, Marcio Oliveira (INPA), Orlando T. Silveira (MPEG), Fernando Z. Vaz de Mello (CEMT), Francisco Limeira de Oliveira (CZMA), Robert Constantin (CCo), Matthias Borer, Isabelle Zürcher (NHMB), Thierry Deuve, Azadeh Taghavian, Antoine Mantilleri (MNHN) and Otto Merkl (HMNH). We are grateful to Marcus Bevilaqua (INPA) and Thiago Polizei (MZUSP) for their help tracing and providing us with specimens for this study, to Adolfo Calor (UFBA) for further information on the type locality of $P$. cleidecostae, to Robert Constantin for comments, suggestions and for providing photographs of type specimens, to Rafael Sousa (MZUSP) for help with image editing. Keita Matsumoto (BMNH) is gratefully acknowledged for the photo of the female of P. flavicornis, and Gabriela Bittencourt-Silva (BMNH) for helpful discussions about Amazonian biogeography. We thank Robert Constantin and Yun Hsiao for the critical reading and suggestions on the manuscript. Part of this work was supported by grants to GB from the São Paulo Research Foundation [FAPESP 2013/08966-1, 2015/21273-0].

\section{CONTRIBUTION OF THE AUTHORS}

Both authors collaborated equally in the conception of the work, species definitions and discussion. GB wrote most of the descriptions and made the identification key, photos and drawings. MG analysed and described specimens from European collections and wrote the discussion about the species distribution.

\section{REFERENCES}

Biffi, G. \& Constantin, R. 2018. Taxonomic revision of Pygodiscodon Wittmer (Coleoptera: Cantharidae: Silinae). Acta Entomologica Musei Nationalis Pragae, 58(1): 77-90. DOI

Blackwelder, R.E. 1945. Checklist of the coleopterous insects of Mexico, Central America, the West Indies and South America. Bulletin United States National Museum, 185(3): 345-550.

Brancucci, M. 1980. Morphologie comparée, évolution et systématique des Cantharidae (Insecta: Coleoptera). Entomologica Basiliensia, 5: 215-388.

Bush, M.B. \& Oliveira, P.E. de. 2006. The rise and fall of the Refugial Hypothesis of Amazonian Speciation: a paleo-ecological perspective. Biota Neotropica, 6(1): bn00106012006 [17 pp]. DOI

Constantin, R. 2010a. A contribution to knowledge of the Cantharidae (Coleoptera, Elateroidea) in Ecuador and French Guiana. Entomologica Basiliensia et Collectionis Frey, 32: 7-29.

Constantin, R. 2010b. Les genres de Cantharidae, Lampyridae, Lycidae et Telegeusidae de Guyane Française (Coleoptera, Elateroidea). Le Coléoptériste, Paris, (Suppl. 2): 32-45.

Constantin, R. 2015. Les Discodon Gorham de Guyane (Coleoptera, Cantharidae). Le Coléoptériste, Paris, (Suppl. 9): 4-34.

Constantin, R. 2017. Les Silinae de Guyane avec la description de quatorze espèces nouvelles (Coleoptera: Cantharidae). Le Coléoptériste, Paris, (Suppl. 11): 41-67.

Constantin, R. \& Chaboo, C.S. 2016. Beetles (Coleoptera) of Peru: A Survey of the Families. Cantharidae Imhoff, 1856. Journal of the Kansas Entomological Society, 89(2): 195-201.

Delkeskamp, K. 1939. Coleopterorum Catalogus, Pars 165, Col. Cantharidae. 's-Gravenhage, W. Junk. 357p.

Delkeskamp, K. 1977. Coleopterorum Catalogus Supplementa, Pars 165, fasc 1. Col. Cantharidae. The Hague, Dr. W. Junk. 485p.

Eberhard, W.G. 2006. Sexually Reversed Copulatory Courtship Roles and Possible Nuptial Feeding in the Soldier Beetle Ditemnus acantholabus (Champion 1915) (Coleoptera: Cantharidae). Journal of the Kansas Entomological Society, 79(1): 13-22.

Hooghiemstra, H. 2001. The continuing debate on the history of the Amazonian rain forest. Amazoniana, 16(3/4): 653-656.

Hsiao, Y.; Hsu, P.-W.; Kuo, S.-Y.; Lee, M.-H. \& Yang, P.-S. 2015. Redescription of Laemoglyptus taihorinensis (Coleoptera: Cantharidae), with Contribution to Female Morphology and Description of Copulation. Acta zoologica bulgarica, 67(2): 193-198.

Knapp, S. \& Mallet, J. 2003. Refuting Refugia? Science, 300(5616): 71-72.

Kury, A.B.; Giupponi, A.P.L. \& Mendes, A.C. 2018. Immolation of Museu Nacional, Rio de Janeiro - unforgettable fire and irreplaceable loss. The Journal of Arachnology, 46(3): 556-559. DOI

Morrone, J.J. 2000. A new regional biogeography of the Amazonian subregion, mainly based on animal taxa. Anales del Instituto de Biología Universidad Nacional Autónoma de México, Serie Zoología, 71(2): 99-123.

Pic, M. 1906. Noms nouveaux et diagnoses de "Cantharini" (Telephorides) européens et exotiques. L'Échange, Revue Linnéenne, 22(264): 90-93.

Vanzolini, P.E \& Williams, E.E. 1970. South American anoles: the geographic differentiation and evolution of the Anolis chrysolepis species group (Sauria, Iguanidae). Arquivos de Zoologia, São Paulo, 19(3-4): 1-298.

Wittmer, W. 1952. 13. Beitrag zur Kenntnis der neotropischen Malacodermata (Coleoptera). Revista Chilena de Entomologia, 2: 197-205.

Wittmer, W. 1966. 27. Beitrag zur Kenntnis der neotropischen Malacodermata (Coleoptera). Studia Entomologica, 9: 411-416. 